\title{
Multiparametric prostate MRI and structured reporting: benefits and challenges in the PI-RADS era
}

\author{
Sanas Mir-Bashiri ${ }^{1} \cdot$ Kaneschka Yaqubi $^{2} \cdot$ Piotr Woźnicki $^{3} \cdot$ Niklas Westhoff $^{4} \cdot$ Jost von Hardenberg ${ }^{4}$. \\ Thomas Huber ${ }^{1,5}$. Matthias F. Froelich ${ }^{5}$. Wieland H. Sommer ${ }^{1,6}$. Maximilian F. Reiser ${ }^{6}$. Stefan O. Schoenberg ${ }^{5}$. \\ Dominik Nörenberg ${ }^{1,5}$
}

Received: 13 July 2020 / Revised: 2 February 2021 / Accepted: 17 February 2021 / Published online: 8 March 2021

(c) The Author(s) 2021

\begin{abstract}
Prostate cancer (PCa) is the second most frequent cancer diagnosis in men and the sixth leading cause of cancer death worldwide with increasing numbers globally. Therefore, differentiated diagnostic imaging and risk-adapted therapeutic approaches are warranted. Multiparametric magnetic resonance imaging (mpMRI) of the prostate supports the diagnosis of PCa and is currently the leading imaging modality for PCa detection, characterization, local staging and image-based therapy planning. Due to the combination of different MRI sequences including functional MRI methods such as diffusion-weighted imaging (DWI) and dynamic contrast-enhanced MRI (DCE-MRI), mpMRI enables a high sensitivity and specificity for the detection of PCa. The rising demand for individualized treatment strategies requires methods to ensure reproducibility, completeness, and quality of prostate MRI report data. The PI-RADS (Prostate Imaging Reporting and Data System) 2.1 classification represents the classification system that is internationally recommended for MRI-based evaluation of clinically significant prostate cancer. PI-RADS facilitates clinical decision-making by providing clear reporting parameters based on clinical evidence and expert consensus. Combined with software-based solutions, structured radiology reports form the backbone to integrate results from radiomics analyses or AI-applications into radiological reports and vice versa. This review provides an overview of imaging methods for PCa detection and local staging while placing special emphasis on mpMRI of the prostate. Furthermore, the article highlights the benefits of software-based structured PCa reporting solutions implementing PI-RADS 2.1 for the integration of structured data into decision support systems, thereby paving the way for workflow automation in radiology.
\end{abstract}

Keywords Prostate cancer $\cdot$ Multiparametric magnetic resonance imaging $\cdot$ Structured reporting $\cdot$ PI-RADS $\cdot$ Radiomics

Sanas Mir-Bashiri and Kaneschka Yaqubi contributed equally.

Dominik Nörenberg

dominik.noerenberg@medma.uni-heidelberg.de

Smart Reporting GmbH, Munich, Germany

2 Department of Clinical Neurobiology, German Cancer Research Center (DKFZ), Heidelberg University, Heidelberg, Germany

3 Experimental Radiation Oncology Group, University of Heidelberg, Heidelberg, Germany

4 Department of Urology, University Medical Center Mannheim, Mannheim, Germany

5 Department of Radiology and Nuclear Medicine, University Medical Center Mannheim, Medical Faculty Mannheim, Heidelberg University, Mannheim, Germany

6 Department of Radiology, University Hospital Munich, Munich, Germany

$\begin{array}{ll}\text { Abbreviations } \\ \text { ADC } & \text { Apparent diffusion coefficient } \\ \text { AFS } & \text { Anterior fibromuscular stroma } \\ \text { AI } & \text { Artificial intelligence } \\ \text { AUC } & \text { Area under the curve } \\ \text { BCR } & \text { Biochemical recurrence } \\ \text { BPH } & \text { Benign prostate hyperplasia } \\ \text { CDSS } & \text { Clinical decision support systems } \\ \text { CT } & \text { Computed tomography } \\ \text { DCE } & \text { Dynamic contrast-enhanced } \\ \text { DRE } & \text { Digital rectal examination } \\ \text { DWI } & \text { Diffusion-weighted imaging } \\ \text { EPE } & \text { Extraprostatic extension } \\ \text { MDSS } & \text { Multifactorial decision support system } \\ \text { MpMRI } & \text { Multiparametric MRI } \\ \text { MRS } & \text { MR spectroscopy } \\ \text { PCa } & \text { Prostate cancer }\end{array}$

Abbreviations

ADC

Apparent diffusion coefficient

Artificial intelligence

Area under the curve

AUC

Biochemical recurrence

$\mathrm{BPH}$

Benign prostate hyperplasia

CDSS

Clinical decision support systems

CT

Computed tomography

DCE

DWI

Diffusion-weighted imagin

Extraprostatic extension

MDSS

$\mathrm{PCa}$

Prostate cancer 


$\begin{array}{ll}\text { PI-RADS } & \text { Prostate imaging reporting and data system } \\ \text { PSA } & \text { Prostate-specific antigen } \\ \text { PSAD } & \text { Prostate-specific antigen density } \\ \text { PSMA } & \text { Prostate-specific membrane antigen } \\ \text { PZ } & \text { Peripheral zone } \\ \text { SVI } & \text { Seminal vesicle invasion } \\ \text { SVM } & \text { Support vector machine } \\ \text { TRUS } & \text { Transrectal ultrasound } \\ \text { TURP } & \text { Transurethral resection of the prostate } \\ \text { T2w } & \text { T2-weighted } \\ \text { TZ } & \text { Transition zone }\end{array}$

\section{Introduction}

Prostate cancer (PCa) is the most commonly diagnosed cancer type in men in the Western world [1], imposing specific requirements for prostate imaging which are essential for the development of risk-adapted therapy approaches. Application of PSA testing in early PCa diagnosis has led to the detection of an increasing number of prognostically favorable cases with low-tumor stages [1]. Nevertheless, there is still a considerable number of highly aggressive primary tumors with possible extraprostatic extension (EPE) and lymph node (LN) or bone/distant metastases in the early stages of the disease.

In the past, the usage of MRI in PCa diagnosis has been limited. Its primary use was restricted to patients with $\mathrm{PCa}$ as an abdominal staging test, and for the evaluation of EPE. Despite adequate anatomic evaluation using morphological MRI sequences, especially for EPE detection in T2-weighted imaging (T2w), MRI showed suboptimal potential for the detection of clinically insignificant PCa [2].

However, the role of MRI in PCa diagnosis has evolved in the last decade: with the addition of novel imaging sequences, such as dynamic contrast-enhanced MRI (DCE), diffusion-weighted imaging (DWI), and MR spectroscopy (MRS), functional information was added to the anatomical image data provided by $\mathrm{T} 2 \mathrm{w}$. Therefore, mpMRI was established as a principle non-invasive imaging technique to support PCa diagnosis, characterization, surveillance, and biopsy guidance. The implementation of a multiparametric MRI protocol led to a migration of PCa detection into earlier stages of the disease providing additional information about tumor aggressiveness in addition to anatomical information [3].

Within this article, a detailed overview of appropriate $\mathrm{PCa}$ detection, structured reporting, and subsequent PCa analysis focusing on an MRI-guided pathway within the course of the disease is given. We discuss the added diagnostic value and clinical significance of mpMRI of the prostate in combination with structured reporting according to the PI-RADS v2.1 classification that, in combination with radiomics analyses, forms the basis for the prospective implementation of artificial intelligence (AI) into the clinical reporting workflow.

\section{Imaging methods for prostate cancer detection}

\section{TNM classification and tumor staging}

TNM classification of PCa is based on the current UICC classification [4, 5]. The tumor stages T1-2 N0 M0 describe locally confined PCa, whereas locally advanced PCa encompasses the stages T3-4 N0/+ and M0. Therefore, locally advanced PCa includes tumors that extend the prostate capsule (T3a), infiltrate the seminal vesicles (T3b) or neighboring structures (e.g., rectum and urinary bladder, T4), or expansions as locoregional $\mathrm{LN}$ metastases $(\mathrm{N}+)$ without distant metastases. Higher Gleason score distribution, a higher proportion of clinical T2 disease (or higher), as well as extraprostatic extension (EPE) and the incidence of perineural invasion are associated with positive surgical resection margins and thus, higher biochemical recurrence rates (BCR) with a worse prognosis, respectively [6]. Within a prospective study investigating 1308 PCa patients, multivariate Cox's proportional hazards regression analysis clearly outlined T3 stages (with presence of ECE) as an independent pathological risk factor for $\mathrm{BCR}$ prediction in comparison to pathological T2 stages $(p<0.001)[6]$.

Clinical practice guidelines propose using the results obtained during primary diagnosis to determine local tumor extent ( $T$ stage). The currently used clinical terminology suggests a differentiation between "locally confined" and "locally advanced" PCa generally referring to DRE without specifying further conditions. Due to increasingly improved therapeutic strategies, medical imaging is of high relevance for the detection of primary PCa as well as for staging of locally advanced PCa considering the presence of EPE, potential lymphogenic or bone/distant metastases [7]. Different approaches for the determination of local tumor extent and the identification of distant metastases exist within the clinical workflow. In addition to DRE and PSA testing, noninvasive imaging techniques such as TRUS, CT, mpMRI are used. Besides that, positron emission tomography (PET) ideally in combination with CT (PET/CT) and mpMRI—can be applied. After bioptic detection of tumor tissue, metastases can be diagnosed through bone scintigraphy, which should be performed in symptomatic patients independent of PSA level, ISUP grade, or clinical stage according to the Guideline Working Panel of the European Association of Urology. In addition, PET/CT has gained increasing clinical relevance because of its added diagnostic value to detect $\mathrm{BCR}$, either as local tumor recurrence or with presence of 
LN or bone metastases $[8,9]$. Gupta et al. demonstrated a superior diagnostic accuracy for the detection of lymph node metastases using ${ }^{68} \mathrm{Ga}$-PSMA PET-CT in comparison to mpMRI with $95.1 \%$ vs. $90.5 \%$, respectively $(p=0.001)$ [9].

\section{Transrectal ultrasonography (TRUS)}

TRUS is used to determine prostate volume and is applied as a first diagnostic test in patients with increased PSA levels to explore PCa localization in addition to its guiding function for systematic or targeted punch biopsies when $\mathrm{PCa}$ is suspected. In prior studies, the diagnostic accuracy to localize PCa was rated significantly inferior in comparison to more advanced imaging modalities such as MRI [10, 11]. A retrospective study of $90 \mathrm{PCa}$ patients demonstrated a significantly higher diagnostic accuracy to detect and especially localize PCa using mpMRI in comparison to TRUS biopsy within the base (AUC 0.81 vs. 0.61 ), mid gland ( 0.75 vs. 0.68$)$, and apex (0.70 vs. 0.67$)$ in a mixed model analysis $(p<0.0001)[11]$.

A high proportion of PCa cannot be detected sufficiently through TRUS due to their heterogenous appearance, with isoechogenic tumors being the most common form on ultrasound imaging. For this reason-and to increase diagnostic accuracy -10 to 12 , systematic core biopsies are considered as standard-of-care, and are recommended by the Guideline Working Panel of the European Association of Urology (EAU) $[10,12]$. The probability of malignancy for a suspicious lesion detected by TRUS varies between 17 and 57\% [13]. Despite these benefits, complications of TRUS-guided prostate biopsies should not be neglected. While the risk for rectal bleeding ranges from 1.3 to $5.8 \%$, inflammation occurs in up to $4 \%$ of patients [12]. Although TRUS enables a volumetric characterization of the prostate as well as the assessment of EPE in some cases, TRUS is not recommended as a staging method for locally advanced PCa by the EAU [10].

\section{Multiparametric magnetic resonance imaging (mpMRI)}

MpMRI describes the combination of anatomic and functional sequences in MRI and is nowadays mainly composed of morphological T2w imaging, DWI, and DCE imaging (i.e., dynamic contrast-enhanced MRI) [14]. The diagnostic value for $\mathrm{PCa}$ detection lies in the variety of possible image contrasts that allow structural imaging and (functional) assessment of healthy and pathologically altered tissue. High-resolution T2w sequences allow the most accurate visualization of the zonal prostate anatomy, the capsule, and assessment of local tumor extension. For the detection of EPE, including seminal vesical invasion (SVI) and infiltration of the neurovascular bundles, $\mathrm{T} 2 \mathrm{w}$ imaging achieves a diagnostic accuracy for the detection of
EPE of up to $0.74[15,16]$. The detection rate of PCa using $\mathrm{T} 2 \mathrm{w}$ sequences is impaired by signal intensity changes based on post-inflammatory, post-therapeutic, and atrophic tissue changes, particularly in the peripheral zone (e.g., in the context of chronic prostatitis). Overall, morphologic $\mathrm{T} 2 \mathrm{w}$ sequences are sensitive (ranging from 88 to $91 \%$ depending on the study) but not specific (ranging from 61 to $67 \%$ ) for the detection of prostate cancer $[14,17,18]$. Combining different MRI techniques including functional MRI methods, DCE-MRI, and DWI leads to higher sensitivity and specificity for the detection of PCa [19-21].

DWI is based on the motility of water molecules within the tissue. Due to the high cell density of tumor tissue, the motility of water molecules is impaired, which enables the differentiation between tumor and normal tissue using DWI. The extent of impaired diffusion can be quantified via apparent diffusion coefficient (ADC) maps (Fig. 1). Thereby, the degree of diffusion restriction allows a qualitative and quantitative assessment of the aggressiveness and clinical significance of PCa. Nowak et al. demonstrated that ADC measures achieved AUC values of up to $78.9 \%$ for discriminating between lesions with Gleason scores $<7$ vs. Gleason scores $\geq 7$ [21].

DCE-MRI (perfusion imaging) consists of multiple fast $\mathrm{T} 1$-weighted $(\mathrm{T} 1 \mathrm{w})$ sequences after a bolus injection of gadolinium-based contrast media. Perfusion imaging allows the examination of contrast agent accumulation within specific areas of the prostate. Perfusion depends on the characteristics of small vessels in the tissue. Since the formation of new vessels (neovascularization) is a common feature of tumors by which their high nutrient requirements are met, tumor tissues show strong blood circulation in these imaging modalities. Thus, PCa may display an early and strong enhancement as well as a fast wash-out of contrast agents (wash-out phenomenon) compared to benign tissue [22-24].

Currently, mpMRI of the prostate is the leading imaging method for the detection, characterization, and assessment of local tumor extent of PCa. If MRI is applied as a first diagnostic test before TRUS-biopsy, it enables the detection of up to $18 \%$ more cases of clinically significant $\mathrm{PCa}$ while simultaneously sparing one in four men the primary biopsy [25]. According to the EAU guidelines [7], mpMRI of the prostate is particularly recommended before biopsies in biopsy naïve patients or for patients with prior negative biopsy and consistent suspicion of PCa (Fig. 2). It reaches high diagnostic accuracy in the detection of PCa especially in anterior regions and areas near the apex of the prostate that can be challenging to detect via TRUS or TRUS-guided biopsies since up to $80 \%$ of lesions identified in the distal apex were shown to be PCa positive at targeted biopsy and may have been missed on TRUS-guided systematic biopsy only $[17,25]$. 

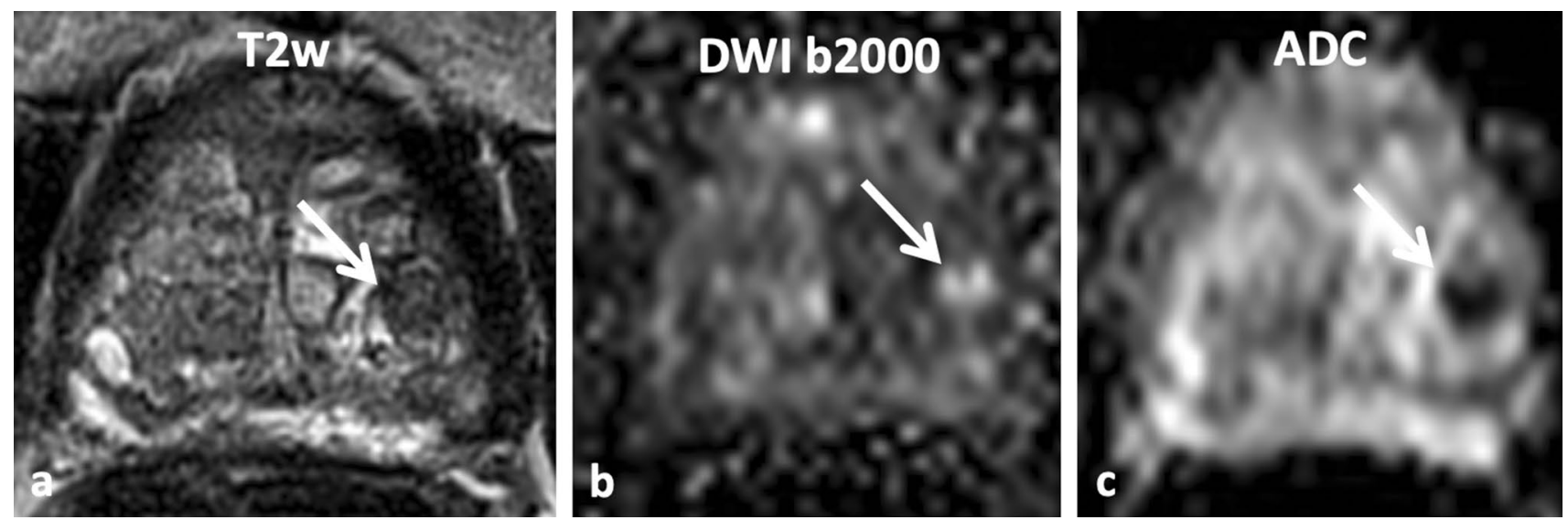

Fig. 1 72-year-old patient with PSA levels of $6.4 \mathrm{ng} / \mathrm{ml}$. a T2whypointense lesion without complete encapsulation (arrow) consistent with a T2w score of 2 (PI-RADS v2.1). b, c The atypical nodular lesion shows strong diffusion restriction including hyperintense signal intensities at high b2000 value images and corresponding low signal

\section{Local tumor staging using mpMRI}

MpMRI is the gold standard for local T-staging of PCa [17]. It is superior to common methods like TRUS and DRE for the assessment of local tumor extent and EPE [18, 26]. A clear advantage of MRI in comparison to other imaging techniques is the possibility of high-resolution depictions of prostate morphology, its capsule, and its neighboring organs. While the transition zone is predominantly evaluated using T2w imaging, the peripheral zone is predominantly evaluated using DWI according to the PI-RADS v2.1 guideline (Table 1). The tumor stage $\mathrm{T} 2$ refers to carcinomas that are restricted to the prostate and do not show signs of EPE (Fig. 3), whereas T3 carcinomas extend beyond the prostate capsule (T3a) or show SVI (T3b), either with continuous tumor spread into the seminal vesicles along the excretory ducts or via secondary invasion of the seminal vesicles after macroscopic EPE [27]. The T4 stage describes a direct tumor infiltration into adjacent organs such as rectum or urinary bladder infiltration.

Although the evaluation of EPE on mpMRI should be interpreted with caution [15], a majority of patients with aggressive tumors, especially intermediate- and high-risk $\mathrm{PCa}$, show a significantly higher risk of capsule infiltration and EPE as assessed within a retrospective study with 1045 PCa patients [16]. Lee et al. evaluated only a limited preoperative staging accuracy of EPE for high pathological stages $(\geq \mathrm{T} 3)$ with an overall sensitivity and specificity of $52.6 \%$ and $82.1 \%$, respectively [16]. Reliable signs of EPE on MRI are the obliteration of the rectoprostatic angle or an infiltration/asymmetry of the neurovascular bundles (T3a). Furthermore, the length of capsular contact has been shown to be more sensitive for the detection of EPE than subjective intensities in ADC maps (arrows in $\mathbf{b}, \mathbf{c}$ ) consistent with a DWI score of 4 (PI-RADS v2.1). The overall PI-RADS score (v2.1) is 3. MRI ultrasound fusion biopsy of this moderately suspicious prostate lesion reveals an acinar adenocarcinoma (Gleason $4+3=7 b$, ISUP grade 3 )

assessments [28-30]. Mehralivand et al. reported that curvilinear contact length to the capsule of more than $1.5 \mathrm{~cm}$ (EPE grade 1), capsular bulge/irregularity (EPE grade 1), both features together (EPE grade 2) or a frank breach of the prostatic capsule with or without invasion of adjacent structures (EPE grade 3) are associated with a higher risk of pathologically confirmed EPE: grade 1 with $24.3 \%$, grade 2 with $38.2 \%$, grade 3 with $66.1 \%$, respectively [28]. EPE of the tumor at the prostate apex with infiltration of the urethral sphincter can also be assessed and is of high clinical relevance since infiltration of the urethral sphincter can lead to urinary incontinence after radical prostatectomy [31].

Assessment of EPE through mpMRI by an experienced radiologist has a negative predictive value of $94-96 \%$ and a positive predictive value of up to $79 \%$ [32-36]. Although a majority of recent studies suggests only moderate sensitivities of $49-57 \%$ for the detection of EPE, mpMRI demonstrated very high specificities of 74-91\% [16, 37, 38]. Sensitivity and specificity for the detection of SVI are comparable with $44 \%$ and $95 \%$, respectively [16]. MRI-based assessment of EPE in the tumor stage pT3 is impaired when no macroscopic signs of periprostatic fat infiltration or obliteration of the rectoprostatic angle are present [39]. Another reason for the low sensitivity of different imaging approaches to detect EPE is the lack of visualization methods of initial microscopic extracapsular extension. Jager et al. demonstrated a significant increase in the detection rate of EPEs from 14 to $100 \%$ depending on the infiltration depth into the extraprostatic fat $(<1 \mathrm{~mm}$ vs. $>3 \mathrm{~mm}$ ) [40].

$\mathrm{LN}$ metastases are present in up to $60 \%$ of patients with locally advanced prostate cancer. However, a meta-analysis of 24 studies [41] demonstrated only a poor sensitivity of $32 \%(22-56 \%)$ and a moderate specificity of $82 \%$ 

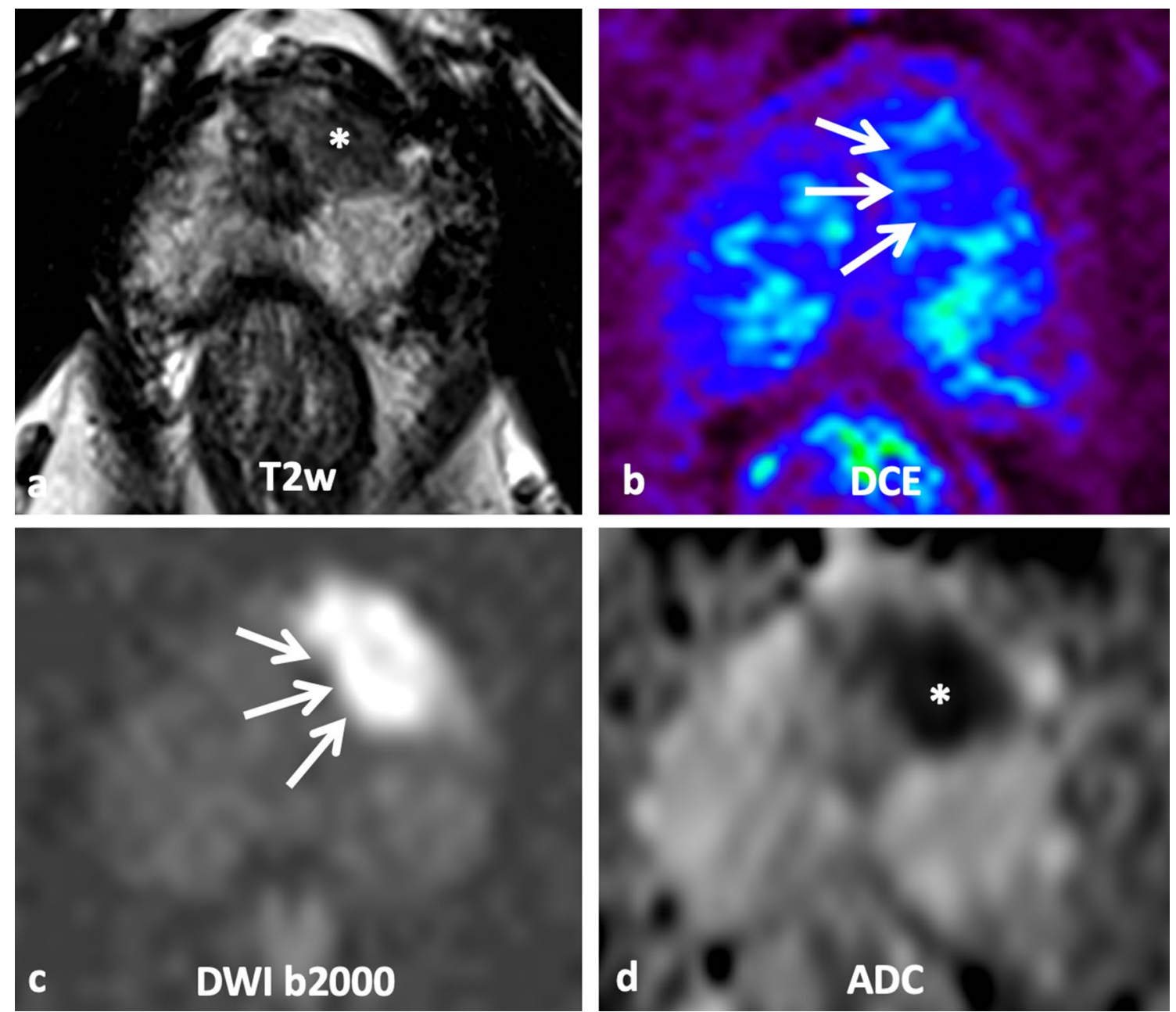

Fig. 2 Prostate cancer in a 54-year-old patient following two consecutive negative TRUS biopsies. a Axial T2w and c, d DWI/ADC images: the tumor (* or arrows), scored as PI-RADS 5 lesion, is localized within the left anterior peripheral zone (PZa) of the apex and shows focal $\mathrm{T} 2 \mathrm{w}$-signal hypointensity with strong diffusion

(79-83\%) for the detection of locoregional LN metastases through mpMRI. Similarly, contrast-enhanced CT showed a poor sensitivity of up to $42 \%$ and a specificity of up to $82 \%$ for the detection of locoregional LN metastases [41]. In contrast, PET/CT, ideally conducted as PSMA-PET/CT, represents one of the most sensitive imaging modalities for the detection of LN (and distant) metastases and is most commonly applied for the post-treatment setting [10, 42]. As highlighted in a recent meta-analysis, PSMA-PET/CT demonstrates a high sensitivity and high specificity of up to $77 \%$ and $97 \%$ for advanced PCa detection in a per-patient analysis [43]. Nevertheless, the detection rate for LN metastases through PSMA-PET/CT in a primary staging setting prior to radical prostatectomy also reveals moderate success rates with sensitivities of $33-65 \%$ and specificities of $98-100 \%$, respectively [44].

restriction b DCE imaging does; however, not exhibit suspicious enhancement dynamics (DCE-). Targeted MRI ultrasound fusion biopsy revealed an acinar adenocarcinoma (Gleason score $4+5=9$, ISUP grade 5)

\section{MRI-guided fusion biopsy}

Ideally, prostate biopsies detect clinically significant $\mathrm{PCa}$ while sparing non-relevant, indolent tumors. Under the current standard-of-care, tissue cores are obtained in a systematical manner through TRUS-guided biopsies, a technique first described by Hodge et al. [45]. However, it was shown to suffer from both poor sensitivity and specificity as a standalone diagnostic test for clinically significant $\mathrm{PCa}$ [25, 46, 47]. Within the PROMIS trial, mpMRI was more accurate than TRUS-biopsy in terms of both sensitivity (93\% vs. $48 \%$ ) and negative predictive value ( $89 \%$ vs. $74 \%$ ) for the detection of clinically significant prostate cancer $(p<0.0001)$ [25].

By combining mpMRI with ultrasound methods as targeted fusion biopsies, these deficits have been overcome 
Table 1 Summary of differences in lesion scoring for PI-RADS v2.1 (2019) vs. PI-RADS v2.0 (2015) for the assessment of clinically significant prostate cancer

Score PI-RADS v2.1

T2WI:transitional zone

T2WI:peripheral zone

DWI: peripheral zone or transitional zone

1 nodule")

3 behavior (normal) tinct margin

3

4 behavior
Normal appearing TZ (rare) or a round, completely encapsulated nodule ("typical

2 A mostly encapsulated nodule or a homogeneous circumscribed nodule without encapsulation ("atypical nodule") or homogeneous mildly hypointense area between nodules

Heterogeneous signal intensity with obscured margins; includes others that do not qualify as 2,4 or 5

4 Lenticular or non-circumscribed, homogeneous, moderately hypointense and $<1.5 \mathrm{~cm}$ in greatest dimension

5 Lenticular or non-circumscribed, homogeneous, moderately hypointense and $\geq 1.5 \mathrm{~cm}$ in greatest dimension or definite extraprostatic extension/invasive

1 Uniform hyperintense signal intensity

2 Linear or wedge-shaped hypointensity or diffuse mild hypointensity, usually indis-

Heterogeneous signal intensity or

Non-circumscribed rounded, moderate hypointensity; includes others that do not qualify as 2,4 or 5

Circumscribed, homogeneous moderate hypointense focus/mass confined to prostate and $<1.5 \mathrm{~cm}$ in greatest dimension

5 Circumscribed, homogeneous moderate hypointense focus/mass confined to prostate and $\geq 1.5 \mathrm{~cm}$ in greatest dimension or definite extraprostatic extension/invasive

No abnormality on ADC, high $b$ value DWI

Linear/wedge-shaped hypointense on ADC and/or

Linear/wedge-shaped hyperintense on high $b$ value DWI

3 Focal (discrete and different from the background) hypointense on ADC and/or

Focal hyperintense on high $b$ value DWI

May be markedly hypointense on ADC or markedly hyperintense on high $b$ value DWI, but not both

$4 \quad$ Focal markedly hypointense on ADC and Markedly hyperintense on high $b$ value DWI

$<1.5 \mathrm{~cm}$ in greatest dimension

$5 \quad$ Focal markedly hypointense on ADC and Markedly hyperintense on high $b$ value DWI

$\geq 1.5 \mathrm{~cm}$ in greatest dimension or definite extraprostatic extension/invasive behavior
PI-RADS v2.0

Homogeneous intermediate signal intensity (normal)

Circumscribed hypointense or heterogeneous encapsulated nodule(s) (BPH)
Indistinct hypointense on ADC

Focal mildly/moderately hypointense on $\mathrm{ADC}$ and

Isointense/mildly hyperintense on high $b$ value DWI 
Table 1 (continued)

Score PI-RADS v2.1

PI-RADS v2.0

DCE-MRI: peripheral zone or transitional zone

\begin{tabular}{|c|c|}
\hline $\begin{array}{l}\text { Focal, and } \\
\text { earlier than or contemporaneously with } \\
\text { enhancement of adjacent normal prostatic } \\
\text { tissues and } \\
\text { corresponds to suspicious finding on } \mathrm{T} 2 \mathrm{w} \\
\text { and/or DWI }\end{array}$ & \\
\hline $\begin{array}{l}\text { No early or contemporaneous enhancement } \\
\text { or } \\
\text { Diffuse multifocal enhancement not cor- } \\
\text { responding to a focal finding on } \mathrm{T} 2 \mathrm{w} \text { and/ } \\
\text { or DWI or } \\
\text { Focal enhancement corresponding to a } \\
\text { lesion demonstrating features of BPH on } \\
\text { T2w (including features of extruded BPH } \\
\text { in the PZ) }\end{array}$ & $\begin{array}{l}\text { No early enhancement or } \\
\text { Diffuse enhancement not corresponding to a } \\
\text { focal finding on } \mathrm{T} 2 \mathrm{w} \text { and/or DWI or } \\
\text { Focal enhancement corresponding to a lesion } \\
\text { demonstrating features of BPH on } \mathrm{T} 2 \mathrm{w}\end{array}$ \\
\hline
\end{tabular}

recently. In addition to implementing a triage test that increases diagnostic accuracy of PCa detection, mpMRI allows to perform mapped and targeted fusion biopsies of suspicious index lesions in addition to systematic but blind samplings [48, 49]. As demonstrated in recent trials, MRITRUS fusion biopsies may prevent up to $27 \%$ of patients from unnecessary biopsies when compared to systematic TRUS-guided biopsies [25]. Kasivisvanathan et al. demonstrated that the detection rate of clinically significant $\mathrm{PCa}$ can be increased with use of targeted biopsies in comparison to standard TRUS-guided biopsies (38\% vs. $26 \%$; $p=0.005$ ) while simultaneously reducing overdiagnosis of clinically insignificant cases (9\% vs. $22 \% ; p<0.001)$ [47].

Diagnostic accuracy of mpMRI can be further improved by including additional clinical parameters such as PIRADS, PSA, prostate volume, age, and DRE results [50]. For instance, performing systematic TRUS-guided biopsies in men with PI-RADS category 1 or 2 will on average detect $8 \%$ of clinically significant PCa cases and at the same time overdiagnose $18 \%$ of patients with insignificant PCa [51]. Therefore, two out of three PCa diagnoses would lead to overtreatment and potential risk of increased patient morbidity. Diagnostic yields for significant PCa increase with higher PI-RADS categories growing from 12 to $21 \%$ over $39-48 \%$ to $72-73 \%$ for PI-RADS categories 3, 4, and 5, respectively [52, 53]. In case of MRI-guided fusion biopsies, these favorable diagnostic results for PI-RADS categories $>3$ are further emphasized even when performing targeted biopsies alone, as this was the case for the abovementioned Precision trial (with $12 \%$ increased detection rates for significant $\mathrm{PCa}$ and $13 \%$ decreased diagnosis of indolent, non-significant tumors) [47]. While some studies suggest that targeted biopsies alone will overlook up to $18 \%$ of cases of clinically significant tumors which would have been detected in systematic TRUS-guided biopsies, most of these additional tumors are often found in sextants adjacent to the lesion identified by mpMRI indicating that factors such as inaccurate sampling and tumor heterogeneity should be taken into account $[49,51]$. In conclusion, evidence is accumulating for multiple benefits of MRI-guided biopsy approaches compared to TRUS-guided biopsies as the standard-of-care. Especially in combination with radiological expertise reflected by structured reporting systems-such as PI-RADS - mpMRI and targeted biopsies do not only perform with significantly increased sensitivity and specificity for the detection of clinically significant $\mathrm{PCa}$, but also reduce the number of men who need to undergo primary biopsies altogether.

\section{Structured reporting and radiomics analysis of prostate cancer}

\section{Report standardization}

The written radiology report is the most common method of communication between radiologists and their stakeholders, who rely on a report with information that will enable clinical decision-making. However, recent studies show gaps in medical imaging report completeness, yet a good quality report is essential for patient care. According to the Institute of Medicine, quality of care can be defined as the degree to which health services for individuals and populations increase the likelihood of desired health outcomes [54]. The radiological report has an impact on all quality dimensions, especially on appropriateness, efficiency, safety, and patient-centered care. A standardized way to communicate reporting results is important to avoid miscommunication, which can be caused by insufficient clinical information provided by the referring physician or a lack of feedback on the 

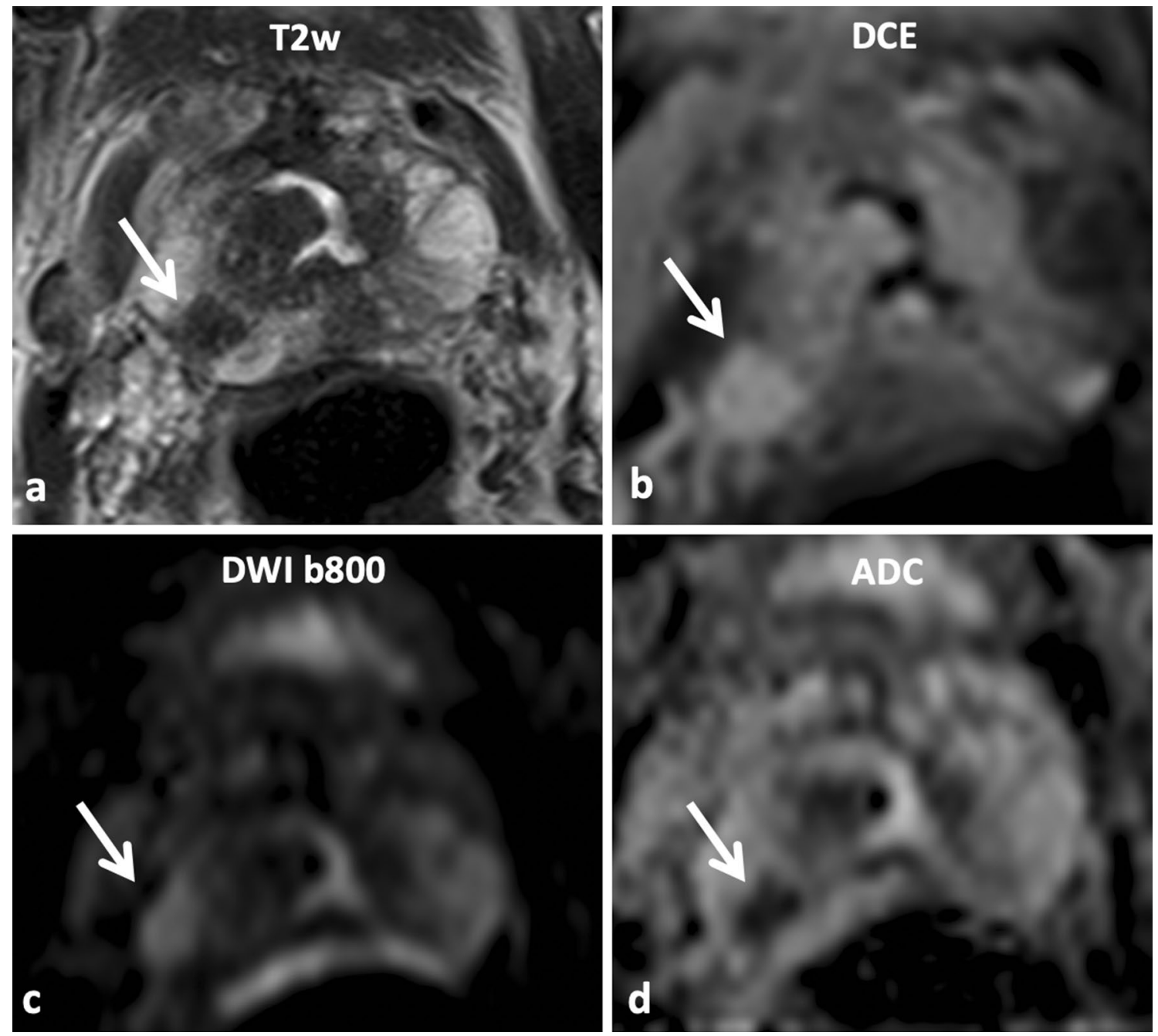

Fig. 3 Prostate cancer in a 78-year-old patient after transurethral resection of the prostate (TURP) and PSA level of $6.3 \mathrm{ng} / \mathrm{ml}$. a Axial $\mathrm{T} 2 \mathrm{w}$ sequence. The tumor is located on the right side within the PZ (mid gland, arrow). b DCE reveals strong early enhancement. c DWI $(b=800)$ and $\mathbf{d}$ ADC map show significant diffusion restriction. The

examination requisition. Structured reporting has developed as a possible solution which narrows the communication quality gap in medicine through standardized integration of feedback into the communication process.

Synoptic reporting depicts the most advanced form of structured reporting since it incorporates discrete fields and fully analyzable data based on radiological ontologies, which in return increase the reproducibility, completeness, and quality of report data for analyses. In a recent survey, $>90 \%$ of radiologists and urologists preferred verbal descriptions of confidence levels and structured prostate MRI reports over free text [55]. Furthermore, structured reporting templates improved the sensitivity of prostate MRI for clinically significant $\mathrm{PCa}$ in the peripheral zone (PZ) from 53 to $70 \%$ [56]. Published documented benefits of structured prostate lesion has a maximal diameter of $1.0 \mathrm{~cm}$ without extracapsular extension revealing an overall PI-RADS score of 4 (v2.1, 2019). MRI ultrasound fusion biopsy demonstrates an acinar adenocarcinoma (Gleason score $4+4=8$, ISUP grade 4 )

MRI reporting include increased perceived clinical impact of report, improved reproducibility, and improved communication $[57,58]$. As such, the use of a specified lexicon to convey ideas such as levels of confidence in the diagnosis of EPE may improve communication and help referring clinicians [59]. Structured reporting encompasses qualitative descriptors based on expertise and has become a priority for many radiological societies, including the European Society of Radiology, the Radiological Society of North America (RSNA), and other organizations such as Cancer Care Ontario [60].

As an example, the RSNA provides RadReport as a webbased reporting application [61] including a prostate MRI reporting template which was constructed based on surveys of radiologists and urologists. For prostate MRI, the 
majority of surveyed radiologists indicated that contrast dose and type, magnetic field strength, coil type, and any medications administered describe essential report components [55, 62]. While these kinds of web-based applications do extract advantages from structured reporting, their restricted integration into existing radiological application systems and missing integration into the workflow at all limits their added value. Additionally, a variety of report solutions vary significantly in levels of complexity and content, rendering them unsuitable for synoptic reporting.

Synoptic reporting requires a solution that enables the integration of structural decision trees based on the input of various stakeholders. By combining structural decision trees with reporting systems, such as PI-RADS, successful communication during the clinical decision-making process can be achieved. Another example of structured radiology reporting are web-based software tools, which interactively transform radiological and clinical findings into structural decision trees (Figs. 4 and 5); therefore, providing the necessary infrastructure for process automation to be integrated into the routine workflow. Along with the improved accessibility and evaluability of clinical data, this facilitates the deployment of radiomics and artificial intelligence to support clinical decision-making [3]. However, successful implementation of advanced software applications requires distinct technical set-ups which cannot be provided in all clinical settings.

\section{PI-RADS version 2.1}

With the rise of MRI usage in PCa diagnosis, characterization, and monitoring, the need for standardized reporting has dramatically increased over the last decade. To address this issue, the European Society of Urogenital Radiology (ESUR) induced the initiative for PI-RADS v1 in 2012, a major structured reporting system for prostate mpMRI preferred by over $80 \%$ of radiologists and urologists [55, 63]. Over the last years, PI-RADS evolved to decrease complexity within the scoring system, define minimum technical parameters for improved result comparability, decrease variability in score interpretations, and augment sensitivity and specificity for the detection of clinically significant PCa.

PI-RADS generates a score from 1 to 5, ranging from very low to very high probability of clinically significant PCa (Fig. 6). In spring 2019, the revised PI-RADS version 2.1 was introduced to resolve limitations which were identified in the previous PI-RADS versions (see Fig. 6 for the summarized PI-RADS v2.1 algorithm). One major focus for the changes of PI-RADS v2.0 to v2.1 lays in adapted diagnostic criteria for lesions in the transition zone (TZ) of the prostate, particularly affecting the assessment of PIRADS category 1-3 lesions (Table 1 ). While PI-RADS v2.0 was able to enhance its accuracy from 81 to $93 \%$ and its sensitivity from 76 to $96 \%$ for TZ lesions compared to PI-RADS v1 [64], diagnosis of TZ lesions remained challenging compared to lesions within the PZ (Table 1). Therefore, more detailed criteria for nodules within the $\mathrm{TZ}$ were introduced in PI-RADS v2.1 leading to improved diagnostic accuracy and reduced interpretation variabilities in this area [3]. For instance, nodules commonly associated with agerelated benign prostatic hyperplasia (BPH) were previously assigned as PI-RADS category 2 lesions, whereas PI-RADS v2.1 now classifies these nodules into PI-RADS category 1 on T2w imaging (Fig. 7a). In addition, PI-RADS v2.1 places special emphasis on DWI scoring to increase the overall PI-RADS category, in particular for the lower T2w imaging categories PI-RADS 2 (Fig. 7b) and PI-RADS 3 (Fig. 6 and Table 1) [3]. PI-RADS v2.0 enabled the upgrade of a T2 $\mathrm{w}$ PI-RADS 3 lesion to a PI-RADS 4 lesion in case of a DWI PI-RADS category of 5. With PI-RADS v2.1, DWI now has the added ability to upgrade a TZ PI-RADS 2 lesion to a PI-RADS 3 lesion in case of a DWI assessment of 4 or 5 [63] (Fig. 1). Following DWI assessment, PZ PI-RADS 3 and 4 lesions may be distinguished utilizing DCE (Fig. 6), which is considered positive if focal early or contemporaneous enhancement is present correlating with a T2 or DWI abnormality (Table 1). In general, an overall suspicion category according to PI-RADS should always be summarized within the radiological report as well as the assignment of a PI-RADS score per lesion. This also accounts for benign focal lesions (such as BPH nodules) as well as for diffuse geographic signal hypointensities which may be classified as (chronic) prostatitis (Fig. 8).

The updated PI-RADS v2.1 classification also includes more information for identifying and classifying cancers in the anterior fibromuscular stroma (AFS) (Fig. 9) as well as in the central zone located as a pyramidal T2 hypointense structure within the prostate base. Especially for these regions, the detection of significant diffusion restriction (ideally using high $b$ values $>1400 \mathrm{~mm}^{2}$ ) as well as suspicious perfusion characteristics of a suspicious lesion on DCE (such as type 2 or type 3 curves) point toward the presence of PCa [63].

A further expansion of PI-RADS v2.1 includes specifications for the use of biparametric MRI over mpMRI, thereby omitting DCE-MRI for selected clinical cases, which provides several benefits: it does not only allow to perform prostate MRI faster and with reduced costs, but also spares the use of contrast agents, thereby avoiding potential gadolinium retentions. However, in some cases, mpMRI is superior to bpMRI due to the improvement of sensitivity provided by DCE-MRI [22-24]. For instance, it was shown that upgrading lesions from PI-RADS category 3 to category 4 based on DCE scores-in this case for PI-RADS v2.0 - resulted in an increased detection of lesions with a Gleason score $\geq 7$ in up to a third of the patients [24]. Consequently, the PI-RADS 

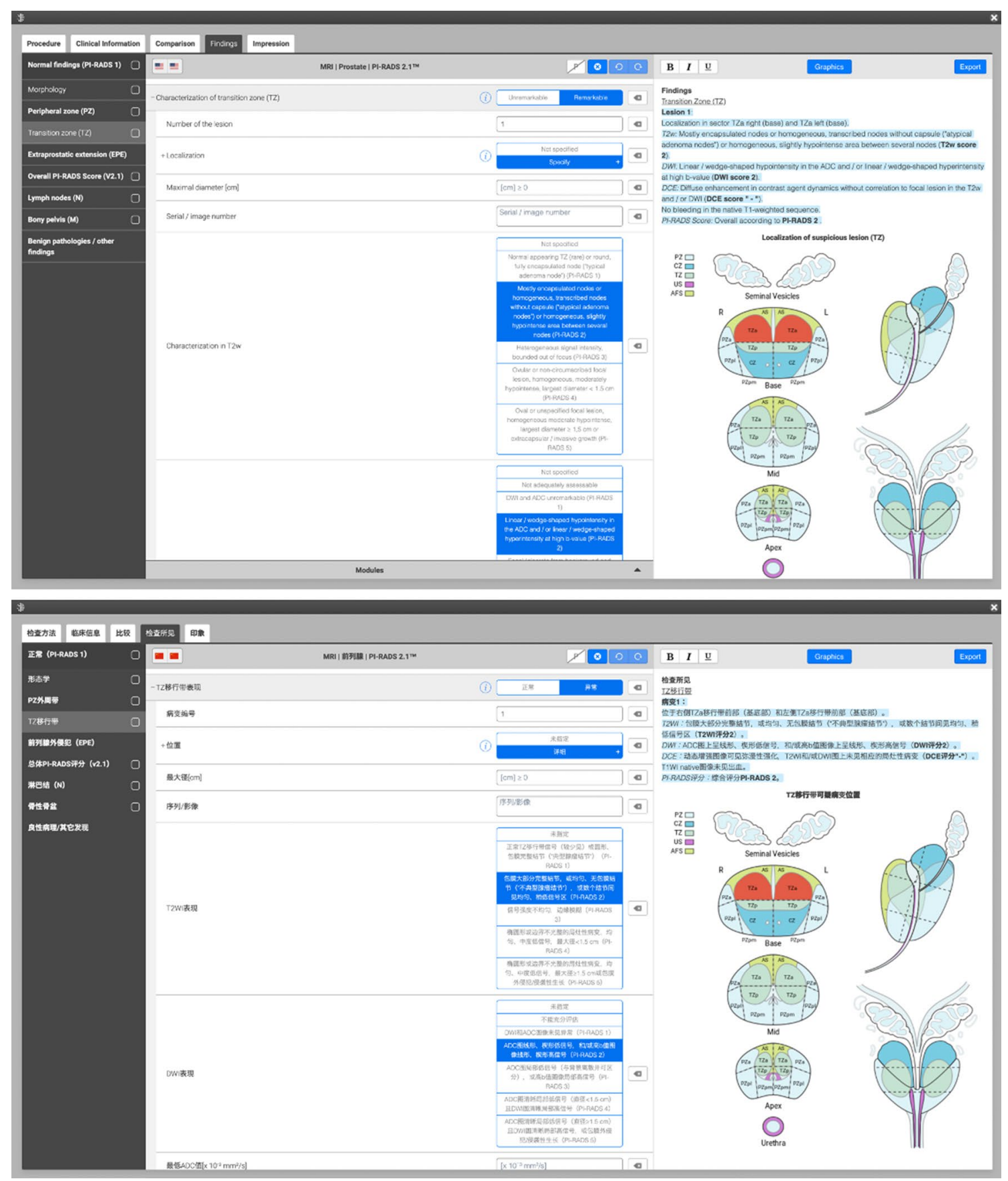

Fig. 4 Structured PI-RADS v2.1 reporting template in English (above) and Chinese (below), exemplarily shown for an interactive web-based software tool which includes a structured decision tree and automatically generated text output with graphical support to enhance interdisciplinary communication and provide clinical decision support committee provides five scenarios for which mpMRI should be preferred in comparison to bpMRI:

1. Patients with prior negative biopsies in combination with unexplained raised serum PSA levels and those in active surveillance under evaluation for fast PSA doubling times or changes in clinical/pathological status.
2. Men with persistent suspicion for harboring clinically significant PCa who have undergone a previous, negative bpMRI. In this case, not missing any clinically significant $\mathrm{PCa}$ has highest priority.

3. Patients with either prior prostate interventions (e.g., TRUS, BPH therapy, radiotherapy) or with prior drug/ hormonal therapies (e.g., testosterone, 5-alpha reduc- 

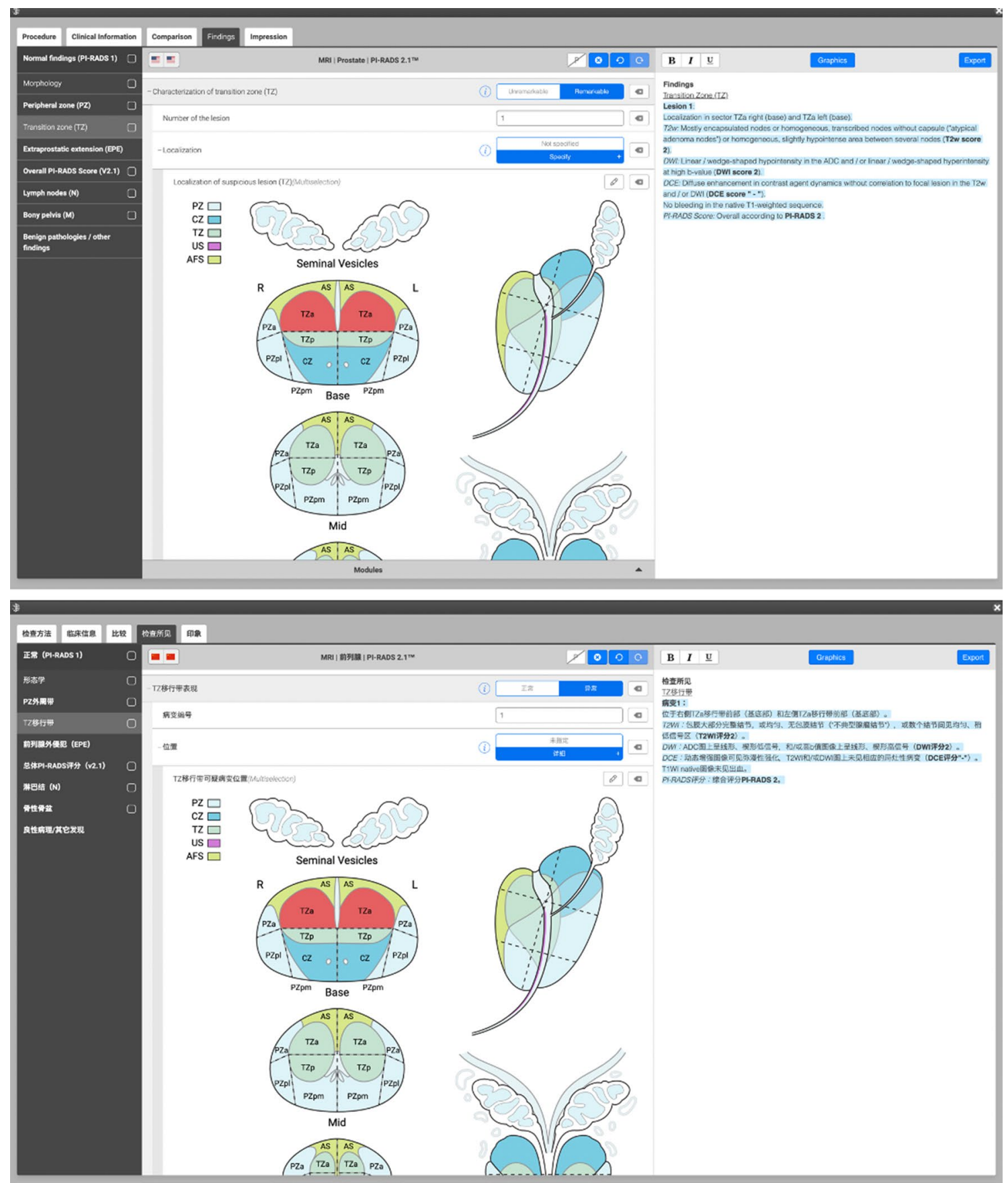

Fig. 5 PI-RADS v2.1 reporting template from an interactive webbased software tool displaying the English (above) and Chinese (below) structured reporting sections. The structured decision tree includes the possibility to select the lesion location from the updated sector map according to PI-RADS v2.1 recommendation, now also including the posteromedial PZ (PZpm) at the base of the prostate as separate sectors tase) which are known for changes in prostate morphology.

4. Biopsy-naïve men with a strong family history, known genetic predispositions, elevated urinary genomic scores, and higher than average risk calculator scores for clinically significant PCa.
5. Patients with a hip implant or other considerations that can be expected to yield degraded DWI.

Other changes in the revised PI-RADS v2.1 include alterations in the sector maps used to compartmentalize the prostate into sub-regions. With the addition of two 
$\mathbf{a}$

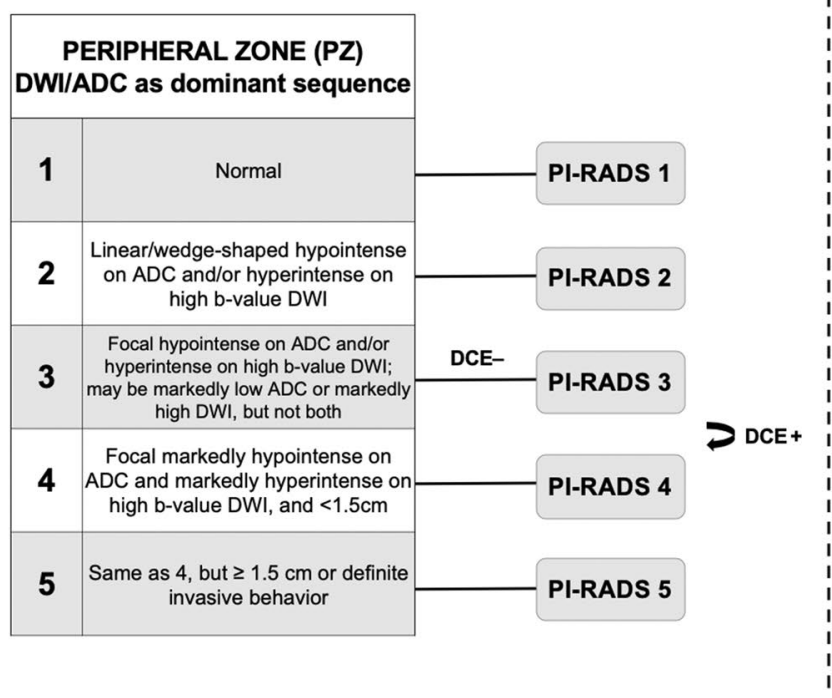

Fig. 6 Simplified PI-RADS v2.1 algorithm for determining the likelihood of clinically significant PCa in a given patient. The PI-RADS system classifies imaging features into five scores with increasing probability of clinically suspicious PCa. This classification relies on DWI as the dominant sequence for the PZ and $\mathrm{T} 2 \mathrm{w}$ imaging for

\section{b}

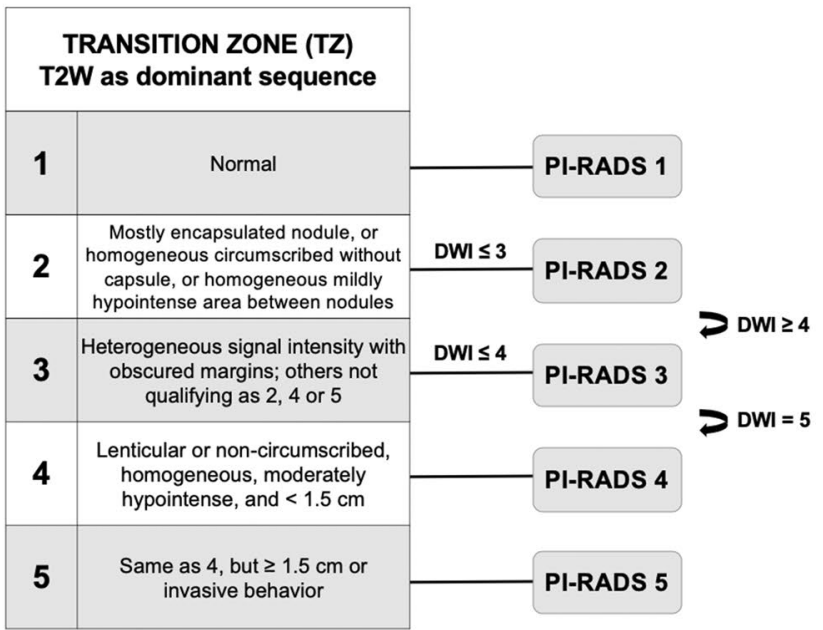

the TZ. Note that positive enhancement in an otherwise PI-RADS 3 lesion of the PZ upgrades that lesion to PI-RADS 4. Similarly, the DWI characteristics of PI-RADS 2 and 3 lesions in the TZ can increase the overall PI-RADS score

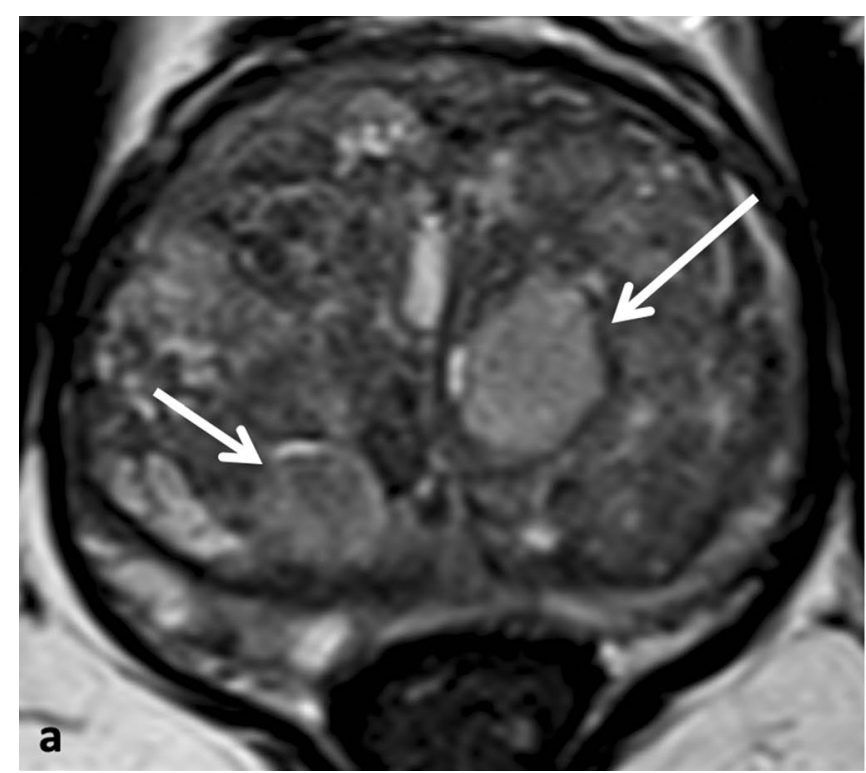

Fig. 7 Enlarged prostate gland of a 71-year-old patient with PSA levels of $15 \mathrm{ng} / \mathrm{ml}$. Axial T2w shows heterogeneous nodular changes in the case of BPH without a suspicious index lesion. a Adenomatous nodules (arrows) exhibit a circular hypointense capsule consistent

regions to the $\mathrm{PZ}$ at the level of the base - the medial right and left posterior PZpm-the sector map is composed of 41 sectors in total (38 within the prostate gland, two corresponding to the seminal vesicles, and one corresponding to the membranous urethra). So far, surveyed practicing

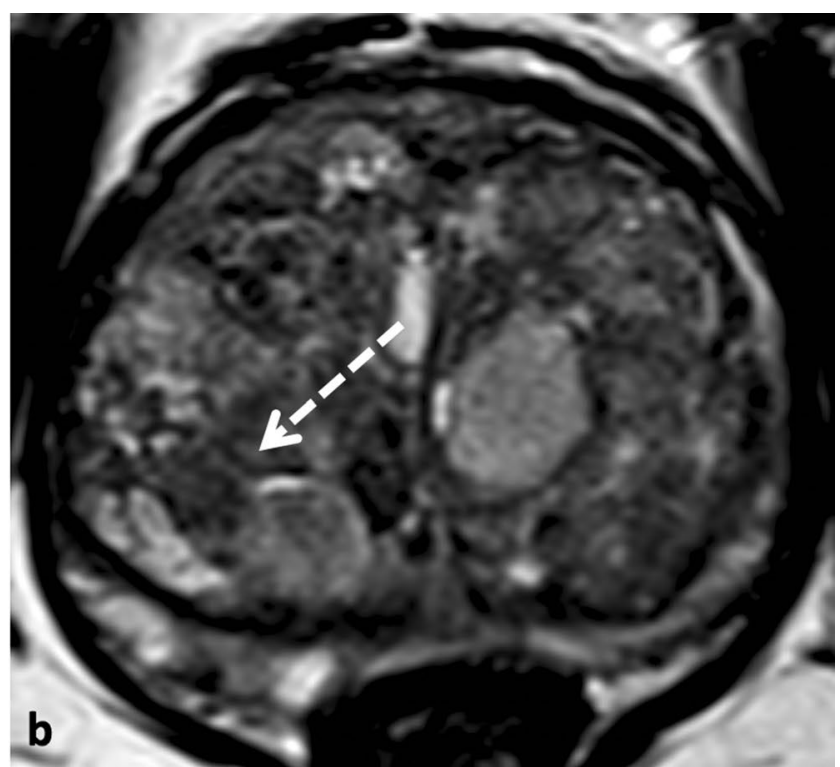

with a PI-RADS score of 1 (v2.1, 2019). b Heterogeneous, hypointense signal attenuation between the adenomatous nodules are in accordance with a PI-RADS score of 2 (dashed arrow). The enlarged $\mathrm{TZ}$ leads to compression of the PZ

radiologists prefer using image series and number, $\mathrm{PZ}$ vs. TZ anatomy, and apex/mid gland/base terminology to localize lesions. Although usage of the PI-RADS sector map is recommended by the PI-RADS committee, it is currently not preferred in all clinical centers, with only 

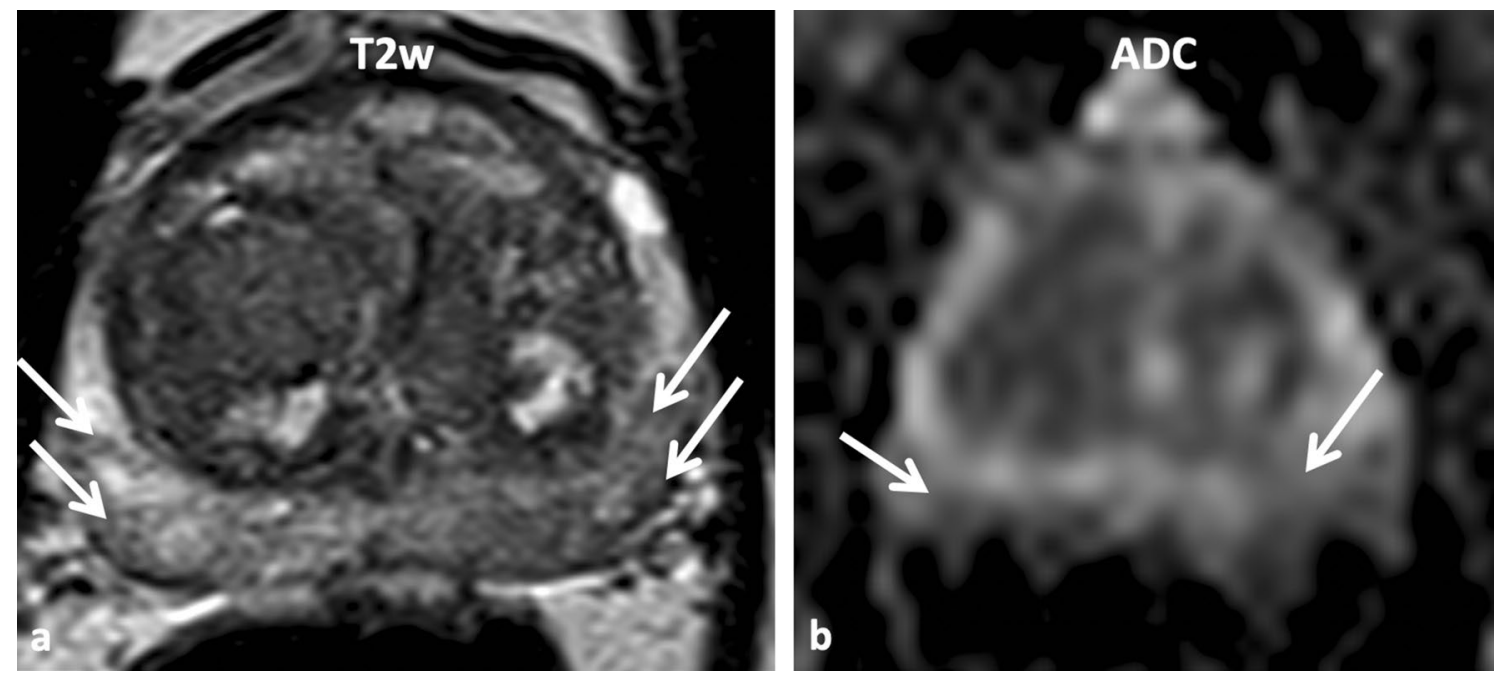

Fig. 8 65-year-old patient with undulating PSA levels between 5 and $7 \mathrm{ng} / \mathrm{ml}$ and clinical suspicion of PCa. a Axial T2w and b ADC map demonstrate bilateral streaky signal hypointensities with non-focal character (arrows) according to PI-RADS 2 (v2.1, 2019). Histology following non-target TRUS biopsy confirms chronic prostatitis, no cancer was detected
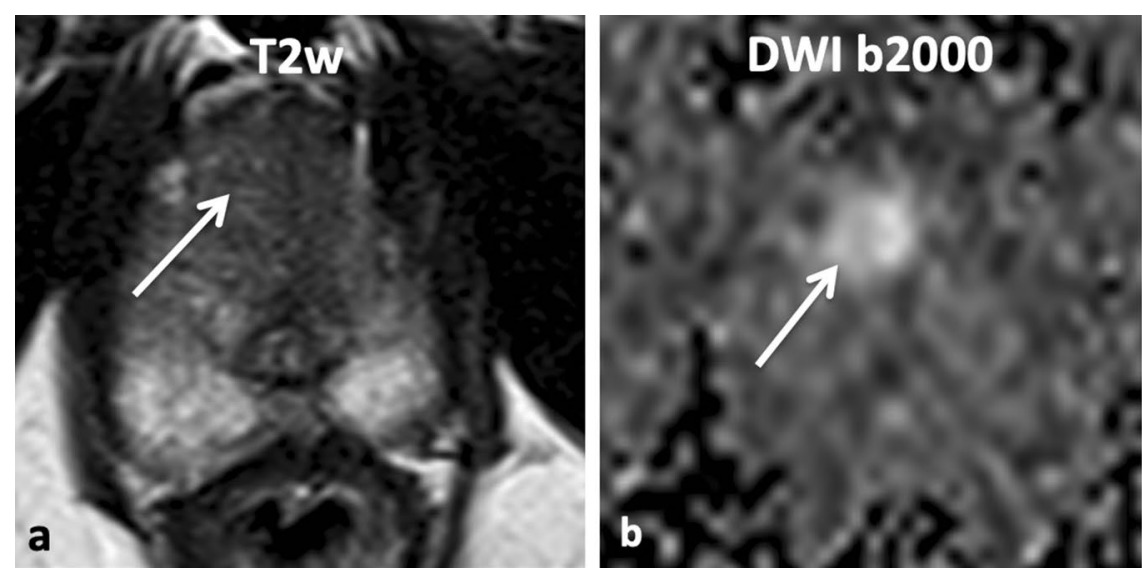

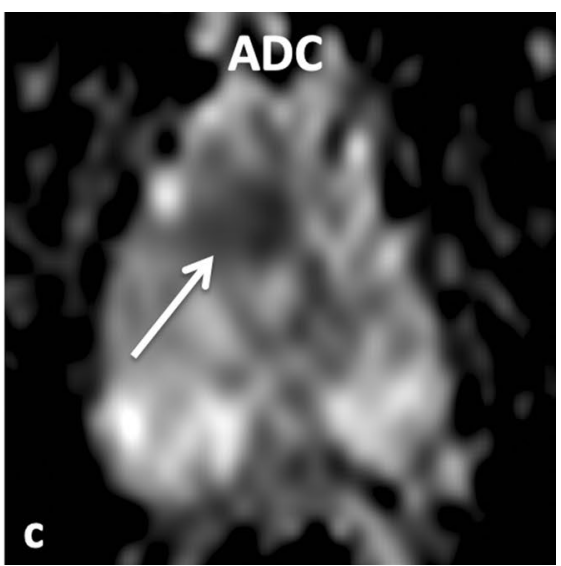

The lesion is scored with a T2w score of 3 and DWI score of 4 . Overall, this leads to a PI-RADS score of 3 (v2.1). MRI ultrasound fusion biopsy of this suspicious lesion reveals an acinar adenocarcinoma (Gleason $3+4=7 \mathrm{a}$, ISUP grade 2). Two prior TRUS biopsies did not detect prostate cancer presence most likely due to its anterior location close to the apex

scoring within the TZ, address the potential use of bpMRI for lesion assessment, revise the sector map, clarify technical features to simplify exam performance, and adjust as well as unify prostate volume measurements. These modifications aim to further strengthen the role of PIRADS scoring in mpMRI, thereby increasing its value in prostate cancer diagnosis, characterization, and clinical decision-making as PI-RADS represents an established semantic biomarker which can be extracted from radiological reports. 


\section{Radiomics}

Radiomics refers to the high-throughput analysis of quantitative image features from standard-of-care medical imaging aiming for prediction of certain clinical markers (clinical survival, radiosensitive phenotypes, or genomic features). This enables data extraction and application in clinical decision support systems by which diagnostic, prognostic, and predictive accuracy is enhanced. As such, the overall aim of radiomics is to reveal disease characteristics that cannot be captured by the naked eye, thereby improving disease diagnostics and treatment. For instance, in the case of PCa detection and classification, it was shown that a radiomics model using extracted features from mpMRI showed superior predictive performance when compared to parameters such as PI-RADS category, mean ADC, or DRE findings. While the radiomics model alone achieved a mean AUC of 0.78, an ensembled radiomics model-which included PI-RADS categories, DRE findings, and PSA density-resulted in an increased mean AUC of 0.89 for the prediction of PCa presence [65]. In order for radiomics to develop, its full potential and to progress as a field from research to clinical routine, a minimum standard of quality regarding image acquisition, image segmentation, feature extraction, and radiomics modeling via machine-learning methods must be achieved. Overall, a whole multitude of factors drastically influences the performance of radiomics-based decision support systems. Thereby, these factors affect outcome predictions, diagnostic accuracy, and therapy planning. In this process, standardization is a crucial step by which robustness and performance of these radiomics-based decision support systems can be achieved [66]. Currently, the field of radiomics lacks standardized evaluation of both scientific integrity and clinical relevance of different radiomics investigations. Thus, distinct evaluation criteria and reporting guidelines need to be established for radiomics to mature as a discipline [66].

Structured reporting of radiological findings describes a powerful tool by which this goal can be achieved. PIRADS incorporates technical specifications and parameters required for standardized imaging. This approach ensures that comparability is given on the level of medical image acquisition itself, enhancing reproducibility and performance of radiomics-based systems. In addition, these structured reporting systems provide access to semantic features, which — when combined with radiomics data—significantly increase the predictive value of radiomics data sets [67].

In summary, structured reporting systems provide a framework by which standardization, accessibility, and completeness of radiological data are increased, thereby paving the way for high-quality data acquisition and complementary radiomics analysis. As such, they yield direct influence on diagnosis, prognosis, and clinical decision-making [56, 66, 67].

\section{Clinical implications}

\section{Role of mpMRI}

Regarding its clinical relevance, mpMRI has become widely accepted as a reliable imaging technique for the detection and diagnosis of PCa. The increasing application of mpMRI in combination with PI-RADS v2.1 intends to prevent unnecessary biopsies in primary diagnostics, as it enables the assessment of suspicious lesions and their local extent. Despite a tendency to underestimate the size of PCa when correlated with histological analysis [68, 69], mpMRI facilitates the conduct of targeted biopsies (e.g., as MRI ultrasound fusion biopsy), which improves pre-therapeutic risk stratification of PCa patients. Assessment of local tumor extent of PCa through mpMRI aids in determining further uroradiological therapeutic concepts. In some European institutions, MRI is used for surgical planning prior to radical prostatectomy. In this case, MRI can facilitate the surgical decision for nerve-sparing surgery to preserve the neurovascular bundles. A recent study assessing positive surgical resection margins within a cohort of 382 patients that underwent radical prostatectomy revealed that TZ tumors were more likely to extend to the apical (37\%) and anterior (22\%) margins, especially in case of bladder neck involvement (37\%), whereas PZ tumors were more likely to extend to the posterolateral (47\%) and also apical (44\%) resection margins, the latter accounted as the most common site of positive surgical margins, respectively [70].

Despite the potential of mpMRI to prove apical positivity as a significant preoperative predictor of a positive surgical margin, image-based evidence of external urethral sphincter infiltration is essential for further surgical planning to maximize the membranous urethra length $[19,31]$.

Standardized application of mpMRI in combination with distinct biopsy procedures allows precise therapy planning for surgical as well as for focal therapeutic procedures (e.g., high-intensity focused ultrasound or cryotherapy) and brachytherapy [71-73]. As part of advanced PCa screenings with a high rate of low-risk cancers, personalized therapy approaches have gained more significance due to lower complication rates when compared to more aggressive therapy approaches [74]. Over the last decades, mpMRI has been increasingly used for radiotherapy planning of locally advanced $\mathrm{PCa}$ in addition to CT to define the exact target volume for radiation treatment. Comparative studies indicate that target volumes for radiation therapy based on MRI findings can be reduced by $30 \%$ as anatomic structures of the apex and posterior gland as well as their surroundings can be determined more precisely in comparison to CT $[75,76]$. The American Urological 
Association and the American Society for Radiation Oncology recommend that adjuvant radiotherapy should be considered for patients with adverse pathologic findings at prostatectomy (i.e., seminal vesicle invasion, positive surgical margins, or EPE) and salvage radiotherapy for patients with PSA or local recurrence after prostatectomy [77]. Hence, the application of mpMRI in the pre-therapeutic setting can support the interdisciplinary confirmation of individualized concepts for patients with locally advanced PCa [78].

\section{Clinical decision support systems for diagnosis of prostate cancer}

Clinical Decision Support Systems (CDSS) offer patientspecific advice based on guideline recommendations to enhance the process of decision-making by physicians and other healthcare practitioners, improve safety, and facilitate evidence-based practice. A multifactorial decision support system (mDSS) describes a tool which improves the clinical decision-making process by utilizing clinical inputs for an individual patient to generate case-specific therapy advice [79].

Different decision support systems can be applied in PCa diagnostics to enhance early tumor detection, reduce overdiagnosis, and avoid unnecessary testing. These systems utilize several parameters including imaging, clinical, and biological features to improve detection and risk classification of $\mathrm{PCa}$ in a minimally invasive way, thereby maximizing individual treatment. mDSS for diagnostic interventions aim to detect, diagnose, or classify PCa using various methods. Several prediction tools are currently used in clinics to support PCa diagnostics including analysis of potential LN involvement, organ confinement, seminal vesicle involvement, and extracapsular extension as well as the risk of failure after treatment [79].

Sadoughi et al. [80] trained an artificial neural network on laboratory results to conduct particle swarm optimization supporting the distinction between localized PCa and benign hyperplasia of the prostate. Van Leeuwen et al. [81] developed a nomogram that includes many parameters, e.g., PIRADS-score, age, PSA, DRE findings, prostate volume, and prior biopsy results. The model performed with an AUC of 0.864 on an external validation set enabling the proposal of an optimal strategy to reduce the number of biopsies needed while minimizing the risk of underdiagnosis. A study conducted by Lee et al. [82] was designed to support the use of a biopsy by predicting initial biopsy outcomes through different models based on TRUS findings and clinical parameters, including age and PSA. Comparing multiple logistic regression analysis, artificial neural networks, and support vector machines (SVM), AUC values of $0.768,0.778$, and 0.847 could be achieved, respectively $[79,82]$.
Due to the application of various MRI sequences in mpMRI, the correct and consistent interpretation of multiparametric image data is still challenging. An automated decision support system that conducts image segmentation, feature extraction, and classification enables a computer-aided diagnosis by which a systematic and objective approach for PCa diagnosis and staging can be achieved [83].

As a comparison between MRI and biopsy results, Chan et al. generated statistical maps based on SVM and Fisher linear discriminant (FLD) analysis on calculated ADC, PD, and $\mathrm{T} 2$ maps from $\mathrm{T} 2 \mathrm{w}$ and DWI performed at $1.5 \mathrm{~T}$ with TRUS-guided biopsy results. Additionally, Vos et al. used $\mathrm{T} 2 \mathrm{w}$ and parametric DCE-MRI maps at 3.0 T validated with whole-mount sections to develop a CAD (Computer-Aided Design) system based on SVM [83].

Another study performed to introduce a new method of decision support systems for detecting and localizing PCa in the PZ is described by Shah et al. [83]. They used SVM to train their model on pathological regions of MRI scans of patients who underwent prostatectomy with the aim of diagnosing PCa as well as locating it specifically on MRI scans by modeling voxel specific risk analyses. Sensitivity and specificity of the model with optimized SVM parameters were both $90 \%$. The generated cancer probability maps can support the localization of tumors and facilitate planning of targeted biopsies and focal therapies [83].

\section{Outlook: workflow automation and artificial intelligence in radiology}

Within healthcare systems, AI will prospectively become a major driver of many applications, including medical diagnostics and imaging, risk management, and hospital management. AI enables the recognition of complex patterns in image data resulting in an automated quantitative assessment. Therefore, more accurate and standardized image assessment is possible when $\mathrm{AI}$ is integrated into the clinical workflow as a tool to assist physicians in their daily routine. The primary trigger for the implementation of AI in medical imaging has been the desire for greater efficacy and efficiency in clinical processes [84].

AI methods are often based on machine learning algorithms. Machine learning involves engineered features that are defined by human experts and interpreted in terms of mathematical equations and can thus be quantified by computer programs. These features are used as inputs to machine learning models, which are trained to classify diseases, thereby supporting clinical decision-making [84]. Machine learning will generate novel algorithms which partly automate several aspects of the radiological workflow including image analysis, interpretation, and report creation to optimize decision support and therapy planning in the long term. 
To achieve this, a workflow integration of quantitative imaging biomarkers detected by AI, radiomics, or structured reporting data is required. Implementing results of quantitative image analysis and image-based measures directly into the radiological report avoids redundant processes and allows to prepopulate radiological reports based on algorithm findings which ensures time-efficient reporting. Prior studies addressing the detection of quantitative imaging biomarkers clearly show an advantage of semi- or fully automated methods of data extraction for outcome prediction [85, 86], the refinement of software tools for importing biomarker data into structured reports [87, 88], the development of tools to facilitate longitudinal imaging biomarker tracking [89], and the integration of these tools into existing radiological information systems [90]. More recent studies show that quantitative imaging biomarkers using radiomicsbased analysis and guideline-driven reporting via PI-RADS can assess tumor aggressiveness and that a combination of clinical factors (such as PSA density), structured reporting (according to PI-RADS) and radiomics analysis even leads to a superior prediction of prostate cancer presence and aggressiveness [65]. Although sufficient scientific evidence is available for a range of radiomics and AI-based studies to enhance tumor characterization and outcome prediction, quantitative image parameters and semantic data from structured reports are nowadays insufficiently incorporated into clinical decision-making processes since the availability of structured reporting software tools as a platform for AI-integration in daily clinical routine is limited. For the widespread dissemination and increasing implementation of practical AI applications in medical diagnostics, huge amounts of data have to be prepared in a structured way and the analyzed models have to be trained continuously [91]. Currently, the dependence of AI on well-curated datasets and large amounts of medical data constitutes a major limitation in the development and advancement of AI algorithms. While in some cases, such as for photographic images, crowdsourcing can be utilized to label and curate a dataset, medical datasets need to be classified by trained personnel to ensure that defined quality criteria are met. Data protection requirements also constrain the development of AI tools and increases both costs and time needed [84].

To overcome these limitations, semi- or fully automated segmentation algorithms have been developed in an effort to curate datasets and prime them for subsequent use with novel AI algorithms. So far, these segmentation algorithms still require human readers for post-hoc verification of the segmentation results, limiting their benefits during the curation process [92]. Combining synoptic reporting and subsequent integration of findings from automated segmentation may overcome these limitations.

Besides, ethical concerns arise when AI is deployed in medicine. AI tools utilize large amounts of sensitive patient data during development and consequent application. To ensure adequate safety, secure connections are required both within medical institutions and to AI systems. So far, major effort has been expended to improve data sharing with minimal safety concerns. For instance, by training AI models with encrypted datasets and sharing the AI models without their input data, AI algorithms can be used without distributing patient data between institutions. 'Cryptonets' further increase privacy, since these deep learning networks are not only trained on encrypted datasets but also provide encrypted predictions which can only be decrypted by the designated institution holding the decryption key [84]. Nonetheless, these solutions are still in their initial development phase and need further advancements before they can be brought into operation in daily routine [60].

\section{Conclusions}

Currently, mpMRI is the leading pre-therapeutic imaging modality for the assessment of local tumor extent of $\mathrm{PCa}$ and superior to other imaging methods such as TRUS. Structured reporting of $\mathrm{PCa}$ is based on the revised PI-RADS classification (v2.1, 2019) which includes modifications in the diagnostic criteria for tumors in the $\mathrm{TZ}$ on $\mathrm{T} 2 \mathrm{w}$, a clear distinction between typical and atypical nodules in the TZ, a revision of the sector map, and clinical indications for the utilization of bpMRI over mpMRI. A workflow integration of radiological results through guideline-driven, softwarebased structured reporting tools and advanced image analysis using radiomics leads to the optimization of diagnostic processes. This may facilitate the implementation of AIbased applications that enable the recognition of complex patterns in image data, resulting in an automated quantitative assessment. Synoptic reporting depicts the most advanced form of structured reporting, since it incorporates discrete fields and fully analyzable data based on radiological ontologies, which increase the reproducibility, completeness, and quality of report data for subsequent analyses building the foundation to standardize and automate the radiological workflow.

Author contributions All coauthors contributed substantially to the success of our research article. SM-B, TH, DN and WS are (part-time) employees of Smart Reporting GmbH (Munich, Germany), KY, PW and MFF are medical students or medical consultants of Smart Reporting GmbH (Munich, Germany) and MFR and SOS are members of the scientific advisory board of Smart Reporting GmbH (Munich, Germany). However, no commercial software or product-related applications have been tested in the study as this is a review article. The research is not financially supported by industry. Academic radiologists (DN, TH, MFF, SOS, MFR) had full control over the academic considerations and outline of the clinical relevance of topics discussed in this research article. There are no other potential conflicts of interests. 
Funding Open Access funding enabled and organized by Projekt DEAL.

Code availability Not applicable.

\section{Declarations}

Conflict of interest Sanas Mir-Bashiri (S.M-B): COI: Employee of Smart Reporting GmbH, Germany. Kaneschka Yaqubi (K.Y.): Department of Clinical Neurobiology, German Cancer Research Center (DKFZ), COI: Working student of Smart Reporting GmbH, Germany. Piotr Woźnicki (P.W.): Experimental Radiation Oncology Group, University of Heidelberg, Germany; COI: Working student of Smart Reporting GmbH, Germany. Niklas Westhoff (N.W.): Department of Urology, University Medical Center Mannheim, Mannheim, Germany; COI: None. Jost von Hardenberg (J.H.): Department of Urology, University Medical Center Mannheim, Mannheim, Germany; COI: None. Thomas Huber (T.H.): Department of Radiology and Nuclear Medicine, University Medical Center Mannheim, Germany; COI: Part-time employee of Smart Reporting GmbH, Germany. Matthias F. Froelich (M.F.F.): Department of Radiology and Nuclear Medicine, University Medical Center Mannheim, Germany; COI: Medical Consultant of Smart Reporting GmbH, Germany. Wieland Sommer (W.S.): Department of Radiology, University Hospital Munich, Germany; COI: CEO of Smart Reporting GmbH, Germany. Maximilian F. Reiser (M.F.R.): Department of Radiology, University Hospital Munich, Germany; COI: Member of scientific advisory board of Smart Reporting GmbH, Germany. Stefan O. Schoenberg (S.O.S.): Department of Radiology and Nuclear Medicine, University Medical Center Mannheim, Germany; COI: Member of scientific advisory board of Smart Reporting $\mathrm{GmbH}$, Germany. Dominik Nörenberg (D.D.): Department of Radiology and Nuclear Medicine, University Medical Center Mannheim, Germany; COI: Part-time employee of Smart Reporting GmbH, Germany.

Availability of data and material Not applicable (review article).

Open Access This article is licensed under a Creative Commons Attribution 4.0 International License, which permits use, sharing, adaptation, distribution and reproduction in any medium or format, as long as you give appropriate credit to the original author(s) and the source, provide a link to the Creative Commons licence, and indicate if changes were made. The images or other third party material in this article are included in the article's Creative Commons licence, unless indicated otherwise in a credit line to the material. If material is not included in the article's Creative Commons licence and your intended use is not permitted by statutory regulation or exceeds the permitted use, you will need to obtain permission directly from the copyright holder. To view a copy of this licence, visit http://creativecommons.org/licenses/by/4.0/.

\section{References}

1. Culp MBB, Soerjomataram I, Efstathiou JA, Bray F, Jemal A. Recent global patterns in prostate cancer incidence and mortality rates. Eur Urol. 2020;77:38-52.

2. Wallis CJD, Haider MA, Nam RK. Role of mpMRI of the prostate in screening for prostate cancer. Transl Androl Urol. 2017;6:464-71.

3. Gupta RT, Mehta KA, Turkbey B, Verma S. PI-RADS: past, present, and future. J Magn Reson Imaging. 2019;52:33-53.
4. O’Sullivan B, Brierley J, Byrd D, Bosman F, Kehoe S, Kossary $\mathrm{C}$, et al. The TNM classification of malignant tumours-towards common understanding and reasonable expectations. Lancet Oncol. 2017;18:849-51.

5. Brierley J, Wittekind C. TNM classification of malignant tumours. 8th ed. New York: Wiley; 2017. p. 191-4.

6. Godoy G, Tareen BU, Lepor H. Site of positive surgical margins influences biochemical recurrence after radical prostatectomy. BJU Int. 2009;104:1610-4.

7. Mottet N, Bellmunt J, Briers E, Bolla M, Bourke L, Cornford P, De Santis M, Henry A, Joniau S, Lam T, Mason MD, Van den Poel H, Van den Kwast TH, Rouvière O, WT members of the E-E-E-SIOG PCGP. EAU-ESTRO_ESUR_SIOG guidelines on prostate cancer. Arnhem: EAU Guidelines Office; 2020.

8. Öbek C, Doğanca T, Demirci E, Ocak M, Kural AR, Yıldırım A, et al. The accuracy of 68Ga-PSMA PET/CT in primary lymph node staging in high-risk prostate cancer. Eur J Nucl Med Mol Imaging. 2017;44:1806-12.

9. Gupta M, Choudhury P, Hazarika D, Rawal S. A comparative study of 68 Gallium-prostate specific membrane antigen positron emission tomography-computed tomography and magnetic resonance imaging for lymph node staging in high risk prostate cancer patients: an initial experience. World J Nucl Med. 2017;16:186.

10. Heidenreich A, Aus G, Bolla M, Joniau S, Matveev VB, Schmid HP, et al. EAU guidelines on prostate cancer. Eur Urol. 2008;53:68-80.

11. Mullerad M, Hricak H, Kuroiwa K, Pucar D, Chen H-N, Kattan MW, et al. Comparison of endorectal magnetic resonance imaging, guided prostate biopsy and digital rectal examination in the preoperative anatomical localization of prostate cancer. J Urol. 2005; 174:2158-63.

12. Harvey CJ, Pilcher J, Richenberg J, Patel U, Frauscher F. Applications of transrectal ultrasound in prostate cancer. Br J Radiol. 2012;85:3-17.

13. Hoogendam A, Buntinx F, de Vet HC. The diagnostic value of digital rectal examination in primary care screening for prostate cancer: a meta-analysis. Fam Pract. 1999;16:621-6.

14. Dickinson L, Ahmed HU, Allen C, Barentsz JO, Carey B, Futterer $\mathrm{JJ}$, et al. Magnetic resonance imaging for the detection, localisation, and characterisation of prostate cancer: recommendations from a European Consensus Meeting. Eur Urol. 2011;59:477-94.

15. Lee T, Hoogenes J, Wright I, Matsumoto ED, Shayegan B. Utility of preoperative 3 Tesla pelvic phased-array multiparametric magnetic resonance imaging in prediction of extracapsular extension and seminal vesicle invasion of prostate cancer and its impact on surgical margin status: experience at a Canadian acad. Can Urol Assoc J. 2017;11:174.

16. Lee H, Kim CK, Park BK, Sung HH, Han DH, Jeon HG, et al. Accuracy of preoperative multiparametric magnetic resonance imaging for prediction of unfavorable pathology in patients with localized prostate cancer undergoing radical prostatectomy. World J Urol. 2017;35:929-34.

17. Rosenkrantz AB, Verma S, Turkbey B. Prostate cancer: top places where tumors hide on multiparametric MRI. Am J Roentgenol. 2015;204:W449-56.

18. Wang L, Hricak H, Kattan MW, Chen H-N, Scardino PT, Kuroiwa K. Prediction of organ-confined prostate cancer: incremental value of MR imaging and MR spectroscopic imaging to staging nomograms. Radiology. 2006;238:597-603.

19. Kenigsberg AP, Tamada T, Rosenkrantz AB, Llukani E, Deng F-M, Melamed J, et al. Multiparametric magnetic resonance imaging identifies significant apical prostate cancers. BJU Int. 2018;121:239-43. https://doi.org/10.1111/bju.13987.

20. Gupta RT, Spilseth B, Patel N, Brown AF, Yu J. Multiparametric prostate MRI: focus on T2-weighted imaging and role in staging of prostate cancer. Abdom Radiol. 2016;41:831-43. 
21. Nowak J, Malzahn U, Baur ADJ, Reichelt U, Franiel T, Hamm B, et al. The value of ADC, T2 signal intensity, and a combination of both parameters to assess Gleason score and primary Gleason grades in patients with known prostate cancer. Acta Radiol. 2016;57:107-14.

22. Greer MD, Shih JH, Lay N, Barrett T, Kayat Bittencourt L, Borofsky S, et al. Validation of the dominant sequence paradigm and role of dynamic contrast-enhanced imaging in PI-RADS version 2. Radiology. 2017;285:859-69.

23. Krishna S, McInnes M, Lim C, Lim R, Hakim SW, Flood TA, et al. Comparison of prostate imaging reporting and data system versions 1 and 2 for the detection of peripheral zone Gleason score $3+4=7$ cancers. Am J Roentgenol. 2017;209:W365-73.

24. Rosenkrantz AB, Babb JS, Taneja SS, Ream JM. Proposed adjustments to PI-RADS version 2 decision rules: impact on prostate cancer detection. Radiology. 2017;283:119-29.

25. Ahmed HU, El-Shater Bosaily A, Brown LC, Gabe R, Kaplan R, Parmar MK, et al. Diagnostic accuracy of multi-parametric MRI and TRUS biopsy in prostate cancer (PROMIS): a paired validating confirmatory study. Lancet. 2017;389:815-22 (The Author(s). Published by Elsevier Ltd. This is an Open Access article under the CC BY license).

26. Wang L, Hricak H, Kattan MW, Schwartz LH, Eberhardt SC, Chen $\mathrm{H}-\mathrm{N}$, et al. Combined endorectal and phased-array MRI in the prediction of pelvic lymph node metastasis in prostate cancer. Am J Roentgenol. 2006;186:743-8.

27. Roethke M, Kaufmann S, Kniess M, Ketelsen D, Claussen $\mathrm{CD}$, Schlemmer HP, et al. Seminal vesicle invasion: accuracy and analysis of infiltration patterns with high-spatial resolution T2-weighted sequences on endorectal magnetic resonance imaging. Urol Int. 2014;92:294-9.

28. Mehralivand S, Shih JH, Harmon S, Smith C, Bloom J, Czarniecki $\mathrm{M}$, et al. A grading system for the assessment of risk of extraprostatic extension of prostate cancer at multiparametric MRI. Radiology. 2019;290:709-19.

29. Rosenkrantz AB, Shanbhogue AK, Wang A, Kong MX, Babb JS, Taneja SS. Length of capsular contact for diagnosing extraprostatic extension on prostate MRI: assessment at an optimal threshold. J Magn Reson Imaging. 2016;43:990-7.

30. Heuck A, Scheidler J, Sommer B, Graser A, Müller-Lisse UG, Maßmann J. MR-Tomographie des Prostatakarzinoms. Radiologe. 2003;43:464-73.

31. Tutolo M, Fossati N, Van der Aa F, Gandaglia G, Montorsi F, Briganti A. Magnetic resonance imaging for membranous urethral length assessment prior to radical prostatectomy: can it really improve prostate cancer management? Eur Urol. 2017;71:379-80.

32. Bittencourt LK, Litjens G, van de Kaa CAH, Turkbey B, Gasparetto EL, Barentsz JO. Prostate cancer: The European Society of urogenital radiology prostate imaging reporting and data system criteria for predicting extraprostatic extension by using 3-T multiparametric MR imaging. Radiology. 2015;276:479-89.

33. Lista F, Gimbernat H, Cáceres F, Rodríguez-Barbero JM, Castillo E, Angulo JC. Evaluación de la invasión extracapsular y otros parámetros de estadificación mediante resonancia nuclear magnética multiparamétrica en pacientes con cáncer de próstata candidatos a prostatectomía radical. Actas Urológicas Españolas AEU. 2014;38:290-7.

34. Soylu FN, Peng Y, Jiang Y, Wang S, Schmid-Tannwald C, Sethi I, et al. Seminal vesicle invasion in prostate cancer: evaluation by using multiparametric endorectal MR imaging. Radiology. 2013;267:797-806.

35. Raskolnikov D, George AK, Rais-Bahrami S, Turkbey B, Shakir NA, Okoro C, et al. Multiparametric magnetic resonance imaging and image-guided biopsy to detect seminal vesicle invasion by prostate cancer. J Endourol. 2014;28:1283-9.
36. Somford DM, Hamoen EH, Fütterer JJ, van Basten JP, van de Kaa $\mathrm{CAH}$, Vreuls W, et al. The predictive value of endorectal 3 Tesla multiparametric magnetic resonance imaging for extraprostatic extension in patients with low, intermediate and high risk prostate cancer. J Urol. 2013;190:1728-34.

37. Raskolnikov D, George AK, Rais-Bahrami S, Turkbey B, Siddiqui MM, Shakir NA, et al. The role of magnetic resonance image guided prostate biopsy in stratifying men for risk of extracapsular extension at radical prostatectomy. J Urol. 2015;194:105-11.

38. de Rooij M, Hamoen EHJ, Witjes JA, Barentsz JO, Rovers MM. Accuracy of magnetic resonance imaging for local staging of prostate cancer: a diagnostic meta-analysis. Eur Urol. 2016;70:233-45.

39. Hricak H, Choyke PL, Eberhardt SC, Leibel SA, Scardino PT. Imaging prostate cancer: a multidisciplinary perspective. Radiology. 2007;243:28-53.

40. Jager GJ, Ruijter ETG, van de Kaa CA, de la Rosette JJ, Oosterhof GON, Thornbury JR, et al. Local staging of prostate cancer with endorectal MR imaging: correlation with histopathology. Am J Roentgenol. 1996;166:845-52.

41. Hövels AM, Heesakkers RAM, Adang EM, Jager GJ, Strum S, Hoogeveen YL, et al. The diagnostic accuracy of CT and MRI in the staging of pelvic lymph nodes in patients with prostate cancer: a meta-analysis. Clin Radiol. 2008;63:387-95.

42. Verburg FA, Pfister D, Heidenreich A, Vogg A, Drude NI, Vöö $S$, et al. Extent of disease in recurrent prostate cancer determined by [68Ga]PSMA-HBED-CC PET/CT in relation to PSA levels, PSA doubling time and Gleason score. Eur J Nucl Med Mol Imaging. 2016;43:397-403.

43. Perera M, Papa N, Roberts M, Williams M, Udovicich C, Vela I, et al. Gallium-68 prostate-specific membrane antigen positron emission tomography in advanced prostate cancer-updated diagnostic utility, sensitivity, specificity, and distribution of prostate-specific membrane antigen-avid lesions: a systematic review and meta-. Eur Urol. 2020;77:403-17. https://doi. org/10.1016/j.eururo.2019.01.049.

44. Grimm M-O, Thomas C, Fröhner M, Wiegel T, Heidenreich A, Thüroff JW, et al. Pelvine Lymphadenektomie und radikale Prostatektomie. Urologe. 2010;49:206-10.

45. Hodge KK, McNeal JE, Stamey TA. Ultrasound guided transrectal core biopsies of the palpably abnormal prostate. J Urol. 1989;142:66-70.

46. Loeb S, Bjurlin MA, Nicholson J, Tammela TL, Penson DF, Carter HB, et al. Overdiagnosis and overtreatment of prostate cancer. Eur Urol. 2014;65:1046-55.

47. Kasivisvanathan V, Rannikko AS, Borghi M, Panebianco V, Mynderse LA, Vaarala MH, et al. MRI-targeted or standard biopsy for prostate-cancer diagnosis. N Engl J Med. 2018;378:1767-77.

48. Marks L, Young S, Natarajan S. MRI-ultrasound fusion for guidance of targeted prostate biopsy. Curr Opin Urol. 2013;23:43-50.

49. Kesch C, Schütz V, Dieffenbacher S, Bonekamp D, Hadaschik BA, Hohenfellner M, et al. Multiparametric MRI fusion-guided biopsy for the diagnosis of prostate cancer. Curr Opin Urol. 2018;28:172-7.

50. Thompson JE, Van Leeuwen PJ, Moses D, Shnier R, Brenner P, Delprado $\mathrm{W}$, et al. The diagnostic performance of multiparametric magnetic resonance imaging to detect significant prostate cancer. J Urol. 2016;195:1428-35.

51. Padhani AR, Barentsz J, Villeirs G, Rosenkrantz AB, Margolis DJ, Turkbey B, et al. PI-RADS Steering Committee: the PI-RADS multiparametric MRI and MRI-directed biopsy pathway. Radiology. 2019;292:464-74.

52. Barkovich EJ, Shankar PR, Westphalen AC. A systematic review of the existing prostate imaging reporting and data system version 2 (PI-RADSv2) literature and subset meta-analysis of PI-RADSv2 
categories stratified by Gleason Scores. Am J Roentgenol. 2019;212:847-54

53. Schoots IG. MRI in early prostate cancer detection: how to manage indeterminate or equivocal PI-RADS 3 lesions? Transl Androl Urol. 2018;7:70-82.

54. Institute of Medicine. Crossing the quality chasm. Washington: National Academies Press; 2001.

55. Spilseth B, Margolis DJ, Patel NU, Ghai S, Rosenkrantz AB, Margolis DJ, et al. A prostate MRI reporting : results from a survey of specialty societies. Am J Roentgenol. 2017 (Epub ahead)

56. Shaish H, Feltus W, Steinman J, Hecht E, Wenske S, Ahmed F. Impact of a structured reporting template on adherence to prostate imaging reporting and data system version 2 and on the diagnostic performance of prostate MRI for clinically significant prostate cancer. J Am Coll Radiol. 2018;15:749-54.

57. Faggioni L, Coppola F, Ferrari R, Neri E, Regge D. Usage of structured reporting in radiological practice: results from an Italian online survey. Eur Radiol. 2017;27:1934-43.

58. Magnetta MJ, Donovan AL, Jacobs BL, Davies BJ, Furlan A, Mj M, et al. Method to optimize prostate MRI. Am J Roentgenol. 2018, pp. 108-12.

59. Wibmer A, Vargas HA, Sosa R, Zheng J, Moskowitz C, Hricak H. Value of a standardized lexicon for reporting levels of diagnostic certainty in prostate MRI. Am J Roentgenol. 2014;203:W651-7.

60. Dobranowski J, Sommer W. Structured radiology reporting: addressing the communication quality gap. SN Compr Clin Med. 2019;1:397-407.

61. Radiological Society of North America. RSNA reporting initiative. [Internet]. www.radreport.org. Accessed 29 Apr 2020

62. Spilseth B, Margolis DJ, Ghai S, Patel NU, Rosenkrantz AB. Radiologists' preferences regarding content of prostate MRI reports: a survey of the society of abdominal radiology. Abdom Radiol. 2018;43:1807-12.

63. Turkbey B, Rosenkrantz AB, Haider MA, Padhani AR, Villeirs G, Macura KJ, et al. Prostate imaging reporting and data system version 2.1: 2019 update of prostate imaging reporting and data system version 2. Eur Urol. 2019;76:340-51. https://doi.org/10.1016/j.eurur o.2019.02.033.

64. Feng Z-Y, Wang L, Min X-D, Wang S-G, Wang G-P, Cai J. Prostate cancer detection with multiparametric magnetic resonance imaging. Chin Med J (Engl). 2016;129:2451-9.

65. Woźnicki P, Westhoff N, Huber T, Riffel P, Froelich MF, Gresser $\mathrm{E}$, et al. Multiparametric MRI for prostate cancer characterization: Combined use of radiomics model with PI-RADS and clinical parameters. Cancers (Basel). 2020;12:1-14.

66. Lambin P, Leijenaar RTH, Deist TM, Peerlings J, de Jong EEC, van Timmeren J, et al. Radiomics: the bridge between medical imaging and personalized medicine. Nat Rev Clin Oncol. 2017;14:749-62.

67. Coroller TP, Bi WL, Huynh E, Abedalthagafi M, Aizer AA, Greenwald NF, et al. Radiographic prediction of meningioma grade by semantic and radiomic features. PLoS ONE. 2017;12:e0187908.

68. Le Nobin J, Rosenkrantz AB, Villers A, Orczyk C, Deng F-M, Melamed J, et al. Image guided focal therapy for magnetic resonance imaging visible prostate cancer: defining a 3-dimensional treatment margin based on magnetic resonance imaging histology co-registration analysis. J Urol. 2015;194:364-70.

69. Priester A, Natarajan S, Khoshnoodi P, Margolis DJ, Raman SS, Reiter RE, et al. Magnetic resonance imaging underestimation of prostate cancer geometry: use of patient specific molds to correlate images with whole mount pathology. J Urol. 2017;197:320-6.

70. O'Neil LM, Walsh S, Cohen RJ, Lee S. Prostate carcinoma with positive margins at radical prostatectomy: role of tumour zonal origin in biochemical recurrence. BJU Int. 2015;116:42-8.

71. van den Bos W, Muller BG, Ahmed H, Bangma CH, Barret E, Crouzet $\mathrm{S}$, et al. Focal therapy in prostate cancer: international multidisciplinary consensus on trial design. Eur Urol. 2014;65:1078-83.
72. Ouzzane A, Betrouni N, Valerio M, Rastinehad A, Colin P, Ploussard G. Focal therapy as primary treatment for localized prostate cancer: definition, needs and future. Future Oncol. 2017;13:727-41.

73. Wang J, Tanderup K, Cunha A, Damato AL, Cohen GN, Kudchadker RJ, et al. Magnetic resonance imaging basics for the prostate brachytherapist. Brachytherapy. 2017;16:715-27.

74. Valerio M, Ahmed HU, Emberton M, Lawrentschuk N, Lazzeri $\mathrm{M}$, Montironi R, et al. The role of focal therapy in the management of localised prostate cancer: a systematic review. Eur Urol. 2014;66:732-51.

75. Krempien RC, Schubert K, Zierhut D, Steckner MC, Treiber M, Harms W, et al. Open low-field magnetic resonance imaging in radiation therapy treatment planning. Int $\mathrm{J}$ Radiat Oncol. 2002;53:1350-60.

76. Sannazzari GL, Ragona R, Ruo Redda MG, Giglioli FR, Isolato G, Guarneri A. CT-MRI image fusion for delineation of volumes in three-dimensional conformal radiation therapy in the treatment of localized prostate cancer. Br J Radiol. 2002;75:603-7.

77. Freedland SJ, Rumble RB, Finelli A, Chen RC, Slovin S, Stein $\mathrm{MN}$, et al. Adjuvant and salvage radiotherapy after prostatectomy: American Society of clinical oncology clinical practice guideline endorsement. J Clin Oncol. 2014;32:3892-8.

78. Mendhiratta N, Taneja SS, Rosenkrantz AB. The role of MRI in prostate cancer diagnosis and management. Future Oncol. 2016;12:2431-43.

79. van Wijk Y, Halilaj I, van Limbergen E, Walsh S, Lutgens L, Lambin $\mathrm{P}$, et al. Decision support systems in prostate cancer treatment: an overview. Biomed Res Int. 2019;2019:1-10.

80. Sadoughi F, Ghaderzadeh M. A hybrid particle swarm and neural network approach for detection of prostate cancer from benign hyperplasia of prostate. Stud Health Technol Inform. 2014;205:481-5.

81. van Leeuwen PJ, Hayen A, Thompson JE, Moses D, Shnier R, Böhm $\mathrm{M}$, et al. A multiparametric magnetic resonance imaging-based risk model to determine the risk of significant prostate cancer prior to biopsy. BJU Int. 2017;120:774-81.

82. Lee HJ, Hwang SI, Han SM, Park SH, Kim SH, Cho JY, et al. Image-based clinical decision support for transrectal ultrasound in the diagnosis of prostate cancer: comparison of multiple logistic regression, artificial neural network, and support vector machine. Eur Radiol. 2010;20:1476-84.

83. Shah V, Turkbey B, Mani H, Pang Y, Pohida T, Merino MJ, et al. Decision support system for localizing prostate cancer based on multiparametric magnetic resonance imaging. Med Phys. 2012;39:4093-103.

84. Hosny A, Parmar C, Quackenbush J, Schwartz LH, Aerts HJWL. Artificial intelligence in radiology. Nat Rev Cancer. 2018;18:500-10.

85. García-Lorenzo D, Francis S, Narayanan S, Arnold DL, Collins DL. Review of automatic segmentation methods of multiple sclerosis white matter lesions on conventional magnetic resonance imaging. Med Image Anal. 2013;17:1-18.

86. Mühlberg A, Holch JW, Heinemann V, Huber T, Moltz J, Maurus $\mathrm{S}$, et al. The relevance of CT-based geometric and radiomics analysis of whole liver tumor burden to predict survival of patients with metastatic colorectal cancer. Eur Radiol. 2020;31:934-46.

87. Pomar-Nadal A, Pérez-Castillo C, Alberich-Bayarri A, García-Martí G, Requena RS, Martí-Bonmatí L. Integrating information about imaging biomarkers into structured radiology reports. Radiologia. 2013;55:188-94. https://doi.org/10.1016/j.rx.2012.11.005.

88. Travis AR, Sevenster M, Ganesh R, Peters JF, Chang PJ. Preferences for structured reporting of measurement data: an institutional survey of medical oncologists, oncology registrars, and radiologists. Acad Radiol. 2014;21:785-96. https://doi.org/10.1016/j.acra.2014.02.008.

89. Abajian AC, Levy M, Rubin DL. Informatics in radiology: Improving clinical work flow through an aim database: a sample web-based lesion tracking application. Radiographics. 2012;32:1543-52. 
90. Abramson RG, Burton KR, Yu JPJ, Scalzetti EM, Yankeelov TE, Rosenkrantz AB, et al. Methods and challenges in quantitative imaging biomarker development. Acad Radiol. 2015;22:25-32. https:// doi.org/10.1016/j.acra.2014.09.001.

91. Pianykh OS, Langs G, Dewey M, Enzmann DR, Herold CJ, Schoenberg SO, et al. Continuous learning AI in radiology: implementation principles and early applications. Radiology. 2020;297:6-14.
92. Sharma N, Ray AK, Shukla KK, Sharma S, Pradhan S, Srivastva A, et al. Automated medical image segmentation techniques. J Med Phys. 2010;35:3-14.

Publisher's Note Springer Nature remains neutral with regard to jurisdictional claims in published maps and institutional affiliations. 